Accepted to ApJ, May 4, 2016

Preprint typeset using IATEX style emulateapj v. 5/2/11

\title{
STAR CLUSTER FORMATION AND DESTRUCTION IN THE MERGING GALAXY NGC 3256
}

\author{
A. J. Mulia ${ }^{1}$, R. Chandar ${ }^{1}$, B. C. Whitmore ${ }^{2}$ \\ Accepted to ApJ, May 4, 2016
}

\begin{abstract}
We use the Advanced Camera for Surveys on the Hubble Space Telescope to study the rich population of young massive star clusters in the main body of NGC 3256, a merging pair of galaxies with a high star formation rate $(\mathrm{SFR})$ and $\mathrm{SFR}$ per unit area $\left(\Sigma_{\mathrm{SFR}}\right)$. These clusters have luminosity and mass functions that follow power laws, $d N / d L \propto L^{\alpha}$ with $\alpha=-2.23 \pm 0.07$, and $d N / d M \propto M^{\beta}$ with $\beta=-1.86 \pm 0.34$ for $\tau<10$ Myr clusters, similar to those found in more quiescent galaxies. The age distribution can be described by $d N / d \tau \propto \tau^{\gamma}$, with $\gamma \approx-0.67 \pm 0.08$ for clusters younger than about a few hundred million years, with no obvious dependence on cluster mass. This is consistent with a picture where $\sim 80 \%$ of the clusters are disrupted each decade in time. We investigate the claim that galaxies with high $\Sigma_{\text {SFR }}$ form clusters more efficiently than quiescent systems by determining the fraction of stars in bound clusters $(\Gamma)$ and the CMF/SFR statistic (CMF is the cluster mass function) for NGC 3256 and comparing the results with those for other galaxies. We find that the CMF/SFR statistic for NGC 3256 agrees well with that found for galaxies with $\Sigma_{\text {SFR }}$ and SFRs that are lower by $1-3$ orders of magnitude, but that estimates for $\Gamma$ are only robust when the same sets of assumptions are applied. Currently, $\Gamma$ values available in the literature have used different sets of assumptions, making it more difficult to compare the results between galaxies.

Subject headings: galaxies: star clusters: general, galaxies: star formation, galaxies: star clusters: individual (NGC 3256)
\end{abstract}

\section{INTRODUCTION}

NGC 3256 is a merging pair of galaxies $\approx 36 \mathrm{Mpc}$ away. Dynamical simulations suggest that the system began interacting $\approx 450$ Myr ago, and it has since undergone a period of major star and star cluster formation. It exhibits two tidal tails that are rich with young massive stellar clusters (Knierman et al. 2003; Maybhate et al. 2007; Trancho et al. 2007a; Mulia, Chandar, \& Whitmore 2015). The main body of NGC 3256 contains a dense population of clusters, many of which are embedded in the galaxy's dusty interstellar medium. The galaxy's intense star formation makes it an extreme environment in which to study cluster formation and destruction.

The cluster population of NGC 3256 has been studied in a number of previous works. Zepf et al. (1999) used $B$ and $I$ band images taken with the Wide Field Planetary Camera 2 on the Hubble Space Telescope (HST) to examine the colors and luminosities of main body clusters. Using the fraction of blue light that they found in clusters, they estimated that the efficiency of cluster formation in NGC 3256 is $\sim 20 \%$. Trancho et al. (2007b) performed optical spectroscopy of 23 clusters in the main body of NGC 3256, finding ages of a few to $\sim 150 \mathrm{Myr}$ and masses from $(2-40) \times 10^{5} M_{\odot}$. Goddard, Bastian, \& Kennicutt (2010; hereafter G10) estimated ages and masses of NGC 3256 clusters from $U B V I$ photometry using $H S T$ 's Advanced Camera for Surveys (ACS) and estimated the fraction of stars forming in bound clusters, hereafter referred to as $\Gamma$, to be $\Gamma=22.9 \%_{-9.8}^{+7.3}$. Their method involved estimating the cluster formation

\footnotetext{
${ }^{1}$ Physics \& Astronomy Department, University of Toledo, Toledo, OH 43606-3390

${ }^{2}$ Space Telescope Science Institute, 3700 San Martin Drive, Baltimore, MD 21218
}

rate (CFR), taken from the total mass of clusters younger than $10 \mathrm{Myr}$, and dividing by the galaxy's star formation rate (SFR).

Some works, including G10, have suggested that there is a correlation between $\Gamma$ and SFR density, $\Sigma_{\text {SFR }}$ (e.g., Silva-Villa \& Larsen 2011; Cook et al. 2012), and between $T_{L}(U)$ (the fraction of $U$ band light contained in clusters) and $\Sigma_{\mathrm{SFR}}$ (Larsen \& Richtler 2000; Adamo Östlin, \& Zackrisson 2011). These relationships imply that the conditions for cluster formation are not universal, but are dependent on conditions in the host galaxy. Chandar, Fall, \& Whitmore (2015), on the other hand, measured the cluster mass function (CMF) in seven galaxies and found that when normalized by the SFR, these CMFs fall nearly on top of one another. The CMF/SFR statistic implies that cluster formation is similar in many galaxies, regardless of SFR. We estimate both $\Gamma$ and, for the first time, the $\mathrm{CMF} / \mathrm{SFR}$ statistic, in NGC 3256.

This paper is arranged as follows. Section 2 describes the observations and cluster selection. Section 3 describes the method for obtaining ages and masses of clusters. Section 4 presents the luminosity functions (LFs), age distributions, and mass functions for NGC 3256. In Section 5, we quantify the effects that distance has on the measured LF and age distribution of the clusters. We determine the $\mathrm{CMF} / \mathrm{SFR}$ statistic and $\Gamma$ from our new NGC 3256 cluster sample in Section 6. We summarize our results and state conclusions in Section 7.

\section{OBSERVATIONS, DATA REDUCTION, AND CLUSTER SELECTION}

\subsection{Observations and Data Reduction}

Our observations come from the ACS on HST. NGC 3256 was observed using the filters $F 555 W(\approx V$ in the 
Johnson-Cousins system; exposed for $2552 \mathrm{~s}), F R 656 N$ $(\mathrm{H} \alpha ; 2552 \mathrm{~s})$, and $F 330 W(\approx U ; 11358 \mathrm{~s})$ as part of the program GO-9735 (PI: Whitmore). The $V$ and $U$ band images were taken in 2003 November using the Wide Field Camera (WFC) and High Resolution Camera (HRC), respectively. The $\mathrm{H} \alpha$ observations were taken in 2004 March. WFC observations using $F 435 W(\approx B)$ and $F 814 W(\approx I)$ filters were taken in 2005 November as part of program GO-10592 (PI: Evans) for 1320 and $760 \mathrm{~s}$, respectively.

The raw data were processed through the standard ACS pipeline. The reduced, multidrizzled WFC images were taken from the Hubble Legacy Archive (HLA) and have a scale of $0.05^{\prime \prime}$ per pixel, while $U$ band images taken with $\mathrm{HRC}$ have $0.025^{\prime \prime}$ per pixel. A $B V I \mathrm{H} \alpha$ color image of NGC 3256 is shown in Figure 1.

\subsection{Cluster Selection}

We use the IRAF3 task DAOFIND on the $I$ band image to make an initial catalog of point sources in NGC 3256. Using the PHOT task in IRAF, we run photometry on the $B, V$, and $I$ band images using an aperture of 3 pixels in radius and a background area between 5 and 8 pixels in radius. For the $U$ band image, we use an aperture radius of 6 pixels and a background area of radius $10-16$ pixels, since the HRC has twice the resolution of the WFC. We use the ACS photometric zeropoint calculator 4 to convert instrumental magnitudes to the VEGAMAG system. We use aperture corrections from 3 pixels to infinity in $B, V$, and $I$ bands taken from isolated star clusters found in the tidal tails of NGC 3256 and reported in Mulia, Chandar, and Whitmore (2015). For the $U$ band aperture correction, we find $\sim 15$ isolated bright clusters and measure their mean $6-20$ pixel magnitude. We use the encircled energy catalog found in Table 4 of Sirianni et al. (2005) for the aperture correction from 20 pixels to infinity. The final corrections were $0.63,0.45,0.42$, and $0.54 \mathrm{mag}$ for $U, B, V$, and $I$ band filters respectively. We also correct the photometry in each filter for foreground extinction, 0.528, 0.441, $0.334,0.264$, and $0.183 \mathrm{mag}$ for $U, B, V, \mathrm{H} \alpha$, and $I$, respectively, taken from the NASA/IPAC Extragalactic Database.

We require photometry in all five $U B V I \mathrm{H} \alpha$ bands in order to accurately age-date star clusters. This has a minimal impact on our catalog and removes only a small fraction $(<10 \%)$ of detected sources. We visually inspect all sources to remove cosmic rays and hot pixels from the catalog.

The low galactic latitude of NGC 3256 means that there will be a significant number of foreground stars from the Milky Way in our images. Luckily, at the distance of NGC 3256, we expect most clusters to be at least partially resolved in the $H S T / \mathrm{WFC}$ images. We measure the concentration index, $C$, to aid our separation of foreground stars and cluster candidates. For a single source, $C$ is measured as the difference in magnitude taken at two different apertures (typically done in

\footnotetext{
3 IRAF is distributed by the National Optical Astronomy Observatory, which is operated by the Association of Universities for Research in Astronomy (AURA) under a cooperative agreement with the National Science Foundation.

${ }^{4}$ http://www.stsci.edu/hst/acs/analysis/zeropoints
}

the $V$ band at 0.5 and 3.0 pixels, which we use here). $C$ has proven to be a robust method in determining the compactness of an object and has therefore often been used to remove stellar contamination from cluster catalogs in many previous works (see Chandar et al. 2010 for a more in-depth discussion on $C$ ). Figure 2 shows $m_{V}$ versus $C$ for all cluster candidates (circles) and likely foreground stars (asterisks). All stars fall to the left of the vertical dashed line at $C=2.2$. We therefore only include cluster candidates with $C>2.2$.

We address incompleteness in our catalog by making a cut where the $m_{V}$ distribution begins to flatten at the faint end, rather than continue in a power law fashion. Because the central portion of NGC 3256 is dustier and has a higher background level than the outer regions, we use separate completeness limits for the inner and outer regions. Clusters in the inner region are complete down to $m_{V} \approx 21.5$, while the outer region is complete down to $m_{V} \approx 23.0$. The separation of these two regions is shown in Figure 11. We find that contamination from background galaxies is negligible in the main body of NGC 3256. In all, we find 505 cluster candidates that meet our selection criteria, and compile their properties in Table 1.

We briefly compare our catalog to that of G10, who studied clusters in the central portion of NGC 3256 using $U V I$ filters taken with the HRC (see Figure1) and the $B$ band taken with the WFC from HST/ACS. Their final sample covered the central $5.8 \times 5.8 \mathrm{kpc}^{2}$ and included 276 clusters after imposing various selection criteria on photometric uncertainty and on the goodness of fit to simple stellar population (SSP) models. The portion of NGC 3256 studied here extends the G10 coverage to include the northern spiral arm, as well as outside the central, brightest portion of the system (as shown in Figure 1). In addition, we include $\mathrm{H} \alpha$ observations in our analysis, which can be critical for breaking the degeneracy between age and extinction, a degeneracy that affects a number of clusters in this merging system (see Section 3.2 for more details). Our catalog includes more than $95 \%$ of the sources that were found by G10, when both catalogs are matched in area and magnitude $\left(m_{V}<21.5\right)$. Conversely, G10 only find $\sim 70 \%$ of the clusters in our catalog (again when matched in area and magnitude). Our study covers an area $\approx 3.5$ times larger than that of G10, although we note a lack of clusters south of the main body.

\section{CLUSTER AGE AND MASS DETERMINATION}

We present the color-color diagram for star clusters in NGC 3256 in Figure 3. The solid line is a stellar population model from G. Bruzual \& S. Charlot (2006, private communication, hereafter BC06; and see also Bruzual \& Charlot 2003) that predicts the evolution of star clusters from about $10^{6}-10^{10} \mathrm{yr}$. All models assume a metallicity of $\mathrm{Z}=0.02$ (i.e., solar metallicity). Numbers mark the logarithmic age $(\tau)$ corresponding to the population.

\subsection{Spectral Energy Distribution (SED) Fitting}

Using $U B V I \mathrm{H} \alpha$ photometry, we fit photometric SEDs of clusters to SEDs from cluster evolution tracks of BC06 in order to determine the age of each cluster. Reddening due to dust can yield inaccurate ages if not accounted 
for, because redder colors imply older ages. We therefore adopt the common technique of varying age and $E(B-$ $V$ ) in order to minimize the function

$$
\chi^{2}(\tau, E(B-V))=\sum_{\lambda} W_{\lambda}\left(m_{\lambda}^{\mathrm{obs}}-m_{\lambda}^{\mathrm{mod}}\right)^{2} .
$$

Here, $m_{\lambda}^{\text {obs }}$ is the observed magnitude and has been corrected for both aperture and foreground extinction (see Section 2.2 for details), and the BC06 models $\left(m_{\lambda}^{\bmod }\right)$ are normalized to each cluster's apparent $V$ band magnitude. The function $W_{\lambda}=\left(\sigma_{\lambda}^{2}+0.05^{2}\right)^{-1}$ weights each photometric measurement in the fit by accounting for the photometric uncertainty $\left(\sigma_{\lambda}\right)$ output by PHOT. We add 0.05 in quadrature to $\sigma_{\lambda}$ to ensure that no single measurement dominates the SED fit.

The BC06 model predicts a mass-to-light ratio (for the $V$ band luminosity) for each age, which we use to estimate a mass for each cluster. $L_{V}$ is calculated from each cluster's $M_{V}$ magnitude (corrected for internal extinction) and the distance modulus of NGC 3256. The masses are computed using a Chabrier (2003) initial mass function.

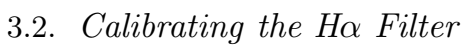

Broad-band colors alone make it difficult to differentiate clusters that are red because they are older from those that are young but appear redder due to the presence of dust. This is known as the age-extinction degeneracy. Including $\mathrm{H} \alpha$ photometry as a fifth point in the SED can help to break this degeneracy and has been used in a number of studies to age-date clusters (e.g., Fall, Chandar, \& Whitmore 2005; Chandar et al. 2010). In this section we describe our method for calibrating the $\mathrm{H} \alpha$ filter in order to use it in the SED fitting method.

We first determine the zero point of the FR656N filter. We identify $\approx 20$ somewhat older star clusters, those that have only continuum emission from stars (i.e., no line emission from ionized gas). We estimate the age and extinction of each of these line-free clusters by performing the $\chi^{2}$ fit described in Section 3.1] using only the $U B V I$ filters to predict a magnitude in the $F R 656 N$ band. Comparison between the predicted and instrumental magnitudes gives a zero point of $22.4 \pm 0.1$. We find that shifting the zero point by 0.1 alters the ages of only $\sim 5 \%$ of the clusters by $\geq 0.3$ in $\log (\tau / \mathrm{yr})$.

Next, we assess whether or not we need to make an adjustment to the predictions for $\mathrm{H} \alpha$ line emission due to the escape of ionizing photons, as has been done in previous work on galaxies at closer distances (e.g., Fall, Chandar, \& Whitmore 2005; Chandar et al. 2010). We compare the predicted and measured $\mathrm{H} \alpha$ maps for a subsample of hand-selected young clusters and find that, unlike for more nearby galaxies, no escape fraction is obviously needed.

In order to understand the impact that the escape fraction $\left(f_{e}\right)$ has on the cluster age estimation, we run the $U B V I \mathrm{H} \alpha$ SED fitting with two values of $f_{e}$; we use the best fit $f_{e}$ of 0.25 , as well as $f_{e}=0.0$. We find that $<5 \%$ of clusters have a difference in age of $\log (\tau / \mathrm{yr})>0.1$ between age estimations from the two escape fractions, and adopt $f_{e}=0.0$ for simplicity due to its negligible impact on age estimates.
We test that cluster ages are reasonable by performing some simple checks. In most galaxies, it has been found that the brightest clusters tend to be young (we discuss the physical implications of this in Section 6.1 e.g., Whitmore et al. 1999). We test our sample by picking the brightest 15 clusters after correction for internal extinction and find that they are all younger than $10 \mathrm{Myr}$. In addition, Figure 4 shows a continuum-subtracted $\mathrm{H} \alpha$ map of NGC 3256, with clusters overlaid that are colorcoded by age. Clusters younger than 10 Myr (blue circles) tend to clump together and follow the brightest $\mathrm{H} \alpha$ regions, while $10-100 \mathrm{Myr}$ old (green) and $100-400$ Myr old (red) old clusters are more sparse and fall on areas with no $\mathrm{H} \alpha$ emission.

\section{RESULTS}

\subsection{Luminosity Function}

We present the LFs for clusters in NGC 3256 in Figure 5] The LF is best described by a power law, $d N / d L \propto L^{\alpha}$, where $\alpha$ is determined from a linear fit to $\log \left(d N / d\left(m_{V}\right)\right)$. The $m_{V}$ values have been aperturecorrected and corrected for foreground extinction in the Galaxy. The three panels of Figure 5 show the LF for the entire sample, as well as for clusters in the inner and outer regions of NGC 3256, separately. We find $\alpha=-2.23 \pm 0.07$ for the combined sample with $m_{V} \leq 21.5$. The LF for the inner and outer regions individually yielded $\alpha \approx-2.1$ in both cases. We also determine the power law index for the combined sample using $B$ and $I$ band magnitudes, and find $\alpha=-2.14 \pm 0.09$ and $\alpha=-2.17 \pm 0.08$, respectively. These are consistent with the $V$ band result of $\alpha=-2.23$, within the uncertainties.

Zepf et al. (1999) previously presented a catalog of clusters in NGC 3256 using poorer quality (in both depth and resolution) $B$ and $I$ band Wide Field Planetary Camera 2 images from $H S T$. Using their cluster catalog to measure the $B$ band LF, we find $\alpha=-2.15 \pm 0.07$, in agreement with the LF presented in this work. In addition, our value for $\alpha$ is similar to that found in many other galaxies (Whitmore et al. 2014).

We also compare the LF found in this work to that of clusters in the eastern tidal tail of NGC 3256, as measured by Mulia, Chandar, \& Whitmore (2015). They obtain $\alpha=-2.61 \pm 0.27$, somewhat steeper than the LF in the main body, for clusters brighter than $M_{V} \approx-8.0$.

\subsection{Age Distribution}

Figure 6] shows the mass-age relation, separated for clusters in the inner and outer regions. The solid lines show our brightness limits of $m_{V}=23.0\left(M_{V}=-9.79\right.$ for a distance modulus of 32.79) for the outer region and $m_{V}=21.5\left(M_{V}=-11.29\right)$ for the inner region. The different completeness limits of the inner and outer regions result in different mass regimes in which the age distribution can be determined. The two dashed lines indicate the masses at which both samples are complete over the age ranges used in the age distribution (Figure 7). The inner cluster sample is complete to $\log (\tau / \mathrm{yr}) \approx 8.0$ for $\log \left(M / M_{\odot}\right)>5.6$, while the outer sample is complete to $\log (\tau /$ yr $) \approx 8.4$ for $\log \left(M / M_{\odot}\right)>5.2$. The apparent lack of clusters in the range $7<\log (\tau /$ yr $)<7.5$ is an artifact of the SED fitting, partially due to the BC06 track forming a loop in color space. 
We find that the age distribution follows a power law with $d N / d \tau \propto \tau^{\gamma}$, with $\gamma \approx-0.67 \pm 0.08$ for two independent mass ranges in both the inner and outer regions. The combined catalog from both regions yields $\gamma \approx-0.5$ for both mass ranges; however, we note that the difference in completeness limits for the two catalogs results in a very low number of clusters for which the age distribution can properly be measured. The age distribution presented in Figure 5 of G10 for clusters in NGC 3256 shows a similar declining shape to that presented here.

Fall \& Chandar (2012) find the cluster age distribution for multiple galaxies to be a power law with $\gamma$ between $\approx-0.6$ and -1.0 . While the slope of the age distribution fluctuates somewhat depending on how the fit is performed, this fluctuation agrees well with the fitting errors shown in Figure 7, as well as with the uncertainties listed in Fall \& Chandar (2012). We conclude that $\gamma$ for NGC 3256 is similar to the values obtained for the cluster populations in other galaxies, although several works find significantly flatter age distributions for clusters in some galaxies (e.g., Hwang \& Lee 2010; Baumgardt et al. 2013; Silva-Villa et al. 2014), including recent work on M31 by the PHAT team (Fouesneau et al. 2014).

The age distribution is a combination of the formation and disruption histories of the clusters. The star formation histories (SFHs) are known independently in a number of galaxies and appear to be relatively constant at least to within a factor of two over the last few hundred million years (e.g., SMC - Harris \& Zaritsky 2004; LMC - Harris \& Zaritsky 2009). Unfortunately, to our knowledge, there is no published $\mathrm{SFH}$ for the merging, starbursting NGC 3256 system, either from direct observations or from high quality simulations. If the $\mathrm{SFH}$ of NGC 3256 has been constant to within a factor of a few over this timescale, as suggested by some simulations for the starbursting Antennae galaxies (Karl, Fall, \& Naab 2011), as well as observational works on interacting galaxies (e.g., Knapen, Cisternas, \& Querejeta 2015 and references therein), then the clusters in NGC 3256 would also have a disruption history similar to that found in these more quiescent galaxies.

\subsection{Mass Function}

We show the CMFs in Figure 8 . These can be described by a power law, $d N / d M \propto M^{\beta}$. We measure $\beta$ for three age ranges for clusters in the inner and outer areas of the galaxy, as well as for the combined sample. The given uncertainties only reflect the formal uncertainty in the fit; we discuss other sources of error in Section 4.3.1.

In the age range $6<\log (\tau / \mathrm{yr})<7$, we find that the combined sample of inner and outer clusters yields $\beta=$ $-1.86 \pm 0.14$. Separating the sample yields $\beta=-1.84 \pm$ 0.14 for outer clusters, but a shallower $\beta=-1.61 \pm$ 0.13 for inner clusters. The somewhat shallower slope is likely a result of crowding, which can artificially flatten the mass function. Studies of other galaxies have also found $\beta \approx-2$ (e.g., Zhang \& Fall 1999; Chandar, Fall, \& Whitmore 2010; Chandar et al. 2010; Chandar, Fall, \& Whitmore 2015). We note that G10 find $\beta=-1.85 \pm 0.12$ for the same age range in NGC 3256, quite similar to our result.

We only determine the mass functions for older clusters in the outer region, since there are very few such clusters in the inner region. We find $\beta=-1.31 \pm 0.36$ in the age range $7<\log (\tau /$ yr $)<8$. This age range is also the most difficult to fit, partially due to the loop in the BC06 track discussed in Section 4.2. Clusters with ages $8<\log (\tau /$ yr $)<8.6$ produce a mass function with $\beta=$ $-2.08 \pm 0.45$, where the large uncertainty in fitting is due to small-number statistics.

In Figure 9, we also show the mass function for NGC 3256 clusters younger than $\log (\tau / \mathrm{yr})=7$ from the $\mathrm{G} 10$ sample. We find that their mass function shows an unexpected systematic offset toward lower masses. The offset is not due to differences in photometry, in SSP model assumptions, or in derived age or extinction values. When we put the G10 photometry through our age-dating procedure, no offset exists. The reason for the offset between the G10 cluster mass estimates and our own is unclear.

\subsubsection{Uncertainties on $\beta$}

Several factors contribute to the uncertainty in the shape of the CMF. We mentioned some of these factors already in Section 3.2, such as the impact that small changes in the zero point and escape fraction for the $\mathrm{H} \alpha$ filter have on $\beta$. In this section, we consider additional contributors to the uncertainties in $\beta$.

Incompleteness can impact $\beta$, causing it to become artificially shallow. This is especially true when considering $\beta$ for a very small age range, where brightness and mass are correlated, and a loss of faint clusters is equivalent to a loss of low-mass clusters. We discussed completeness of our catalog in Section 2.2, and we choose selection limits that are sufficiently bright that the impact of incompleteness is minimal.

Details of the fitting procedure can also affect $\beta$. We find that the specific mass range used in the fits affects $\beta$ by less than about 0.05 for samples with 40 or more clusters, similar to most of the subsets quoted in this paper. We also measure the impact of bin size on $\beta$ and again find $\beta$ to fluctuate by \pm 0.05 . Overall we find that all of these sources of uncertainty affect $\beta$ within the formal uncertainty given in Figure 8 for each age range.

\subsubsection{Is There an Upper Mass Cutoff?}

Some works have found that mass functions for clusters in spiral galaxies deviate from a single power law and have a downturn at the high-mass end (e.g. Bastian et al. 2012). These works suggest that the mass function is better described by a Schechter function, $d N / d M \propto M^{\beta} \exp \left(-M / M_{C}\right)$, where $M_{C}$ is the upper mass cutoff. While the lower-mass end of the Schechter function converges to a power law with index $\beta$, the highmass end drops off from a power law quickly above $M_{C}$. In NGC 3256, we find that a single power law provides a good fit to the observed CMF, since no obvious downturn is observed at the high-mass end. Therefore, our distribution is only consistent with a Schechter function with a high $M_{C}$ (consistent with the suggestion that any $M_{C}$ would be expected to be higher than $10^{6} M_{\odot}$ for this type of system; e.g., Kruijssen 2014). Below, we determine a lower limit to $M_{C}$. For simplicity, we compare our CMFs for $6<\log (\tau /$ yr $)<7$ and $8<\log (\tau /$ yr $)<8.6$ to Schechter functions with a fixed $\beta=-2.0$ and varying $M_{C}$ values, although there is no reason why $\beta$ has to be exactly -2.0 . For the latter age range, we use only the sample taken from the outer region of the central galaxy due to low-number statistics. 
Using this approach, the mass function of clusters with ages $6<\log (\tau / \mathrm{yr})<7$ can only be reasonably fit for a Schechter function with $\log \left(M_{C} / M_{\odot}\right)$ values above 6.5. Because the mass function is fit out to $\log \left(M / M_{\odot}\right) \sim$ 5.75 , it is not surprising that Schechter functions with $\log \left(M_{C} / M_{\odot}\right)<6.5$ begin to fall below the observed mass function at the high-mass end. The left panel of Figure 10 shows the mass function with multiple $M_{C}$ values.

Fitting the mass function for $8<\log (\tau /$ yr $)<8.6$ with a Schechter function is indistinguishable from fitting a pure power law for $\log \left(M_{C} / M_{\odot}\right) \geq 6.5$. This is shown in the right panel of Figure 10, where $\log \left(M_{C} / M_{\odot}\right)=6.0$ and $\log \left(M_{C} / M_{\odot}\right)=6.5$ provide reasonable fits to the mass function, as does a single power law. The mass function is fit out to $\log \left(M / M_{\odot}\right) \sim 5.5$, and we find that the Schechter functions with $\log \left(M_{C} / M_{\odot}\right)<6.0$ begin to fall below the observed mass function at the high end.

We find little evidence for a truncation of the mass function at the high end. We conclude that while both mass functions tested here can be fit with a Schechter function, it is not required and that a single power law provides an equally good fit to each. We therefore favor a pure power law in fitting the mass functions.

\section{HOW DOES DISTANCE AFFECT THE OBSERVED CLUSTER DISTRIBUTIONS?}

The specific method used to select star clusters, particularly at ages $\tau<10 \mathrm{Myr}$, may affect the results (e.g., Bastian et al. 2012). In addition, cluster selection in galaxies located at different distances will necessarily be limited by resolution since at least some sources will appear as diffuse stellar clusters in nearby galaxies but not in more distant ones.

At a distance of $36 \mathrm{Mpc}$, NGC 3256 is nearly twice as far as the Antennae, the nearest example of a pair of actively merging galaxies. In order to assess how distance might impact source selection, as well as the LFs, mass functions, and age distributions of cluster populations in NGC 3256, we use the well studied HST images of the Antennae, and compare the resulting cluster distributions when we simulate the entire system to lie a factor of two further away.

We download the Antennae data from $H S T / W F C$ $F 435 W, F 550 M$, and $F 814 W$ filters, as well as the WFC $3 F 336 \mathrm{~W}$ filter from the HLA. The combined image for each filter is then boxcar-smoothed with a kernel width of two pixels. We run DAOFIND on both the original and degraded $F 550 M$ images, tuning only the FWHM parameter in both images, and we detect 8405 and 3173 objects, respectively. We refer to the cluster catalogs produced from the unaltered and degraded images as the "original" and "image-smoothed" catalogs, respectively. We perform aperture photometry on all detected sources and correct for foreground extinction. In addition, we measure and apply separate aperture corrections to each catalog.

Figure 11 shows a comparison of the original and image-smoothed F550M images. The circles indicate detections from DAOFIND. It is evident that the imagesmoothed source catalog is missing many of the very faint clusters in addition to some of the bright sources. We illustrate this in Figure 12, where the bottom panel shows the relative fraction of sources found in the image- smoothed catalog compared to the original catalog 5 We find that $\approx 85 \%$ of the 3173 image-smoothed detections share coordinates (within one pixel) with their original image counterparts.

The top and middle panels of Figure 12 show the LFs for the original and image-smoothed cluster catalogs, respectively. Both LFs are fit for $m_{V}<22.7\left(M_{V}<-9\right)$, where the $m_{V}$ distribution begins to flatten out at the faint end. We find values for $\alpha$ of $-2.13 \pm 0.04$ for the clusters detected in the original image, very similar to those found in previous works. For the smoothed image sample we also find $\alpha=-2.10 \pm 0.04$. A similar and more extensive study was performed by Randriamanakoto et al. (2013), where it was also determined that $\alpha$ is not greatly impacted by resolution. They performed various convolutions on their image in order to simulate different galaxy distances, finding that $\alpha$ differed by no more than 0.2 .

The color distributions of clusters brighter than $M_{V}<$ -9 from the original and smoothed images are quite similar. While there are more points from the original catalog present in Figure 13, the scatter in the colors among the two catalogs is roughly the same. We run SED fitting on both catalogs and construct their age distributions (not shown), finding $\gamma \approx-0.8$ in both cases.

We find that the LF, color distributions, and age distributions for the original and image-smoothed catalogs are nearly the same, and we therefore conclude that distance does not significantly hamper our ability to study clusters out to $\approx 40 \mathrm{Mpc}$.

\section{HOW EFFICIENTLY DOES NGC 3256 FORM CLUSTERS?}

Many previous works have suggested that galaxies with a high SFR per unit area $\left(\Sigma_{\mathrm{SFR}}\right)$ form clusters more efficiently than galaxies with lower $\Sigma_{\text {SFR }}$ (e.g., G10; Kruijssen 2012; Kruijssen \& Bastian 2016). Two independent observational methods have been developed to test this: the CMF/SFR statistic (Chandar, Fall, \& Whitmore 2015; hereafter CFW15) and $\Gamma$, the fraction of stars formed in bound clusters (Bastian et al. 2008). A third, less direct method, $T_{L}(U)$, has also been used (Larsen \& Richtler 2000). In this work, we focus on the first two methods, because they are easier to interpret. In this section, we apply both methods to our cluster sample in NGC 3256, and compare the results with those from previous works and for different galaxies.

\section{1. $C M F / S F R$ Statistic}

CFW15 compared the CMFs between several different galaxies by normalizing them by the SFR of the host galaxy, and comparing the amplitudes of the resulting CMF/SFR statistic. The sample included spirals (M83 and M51), irregulars and starbursts (Large and Small Magellanic Clouds, NGC 4214, and NGC 4449), and the merging Antennae galaxies. The large range of amplitudes for the CMFs in two different ranges of age $(\log (\tau /$ yr $)<7$ and $8<\log (\tau /$ yr $)<8.6)$ in these galaxies collapse when divided by their respective SFRs. The

5 We do not restrict our sample to objects that appear to be spherically symmetric, as has been recommended to separate bound from unbound clusters (e.g., Gieles \& Portegies Zwart 2011), since resolution makes this difficult at the distance of the Antennae and NGC 3256. 
dispersion in the $\mathrm{CMF} / \mathrm{SFR}$ statistic is only $\sigma \approx 0.2$, similar to the expected uncertainties. Since the CMF/SFR statistic of very young clusters is similar across these different galaxies, CFW15 concluded that cluster formation scales with the overall SFR on galaxy scales. The similarity of the CMF/SFR statistic for the older clusters suggests that the disruption histories are also similar across the galaxies.

Here, we determine the CMF/SFR statistic for NGC 3256 for the first time and compare with the rest of the galaxies in the CFW15 sample. The mass functions discussed in Section 4.3 were shown to follow a power law with index $\beta$. In the left panel of Figure 14, we show the unnormalized CMFs for the same seven galaxies shown in CFW15 and now also include NGC 3256. The CMF for NGC 3256 is higher than that of the other galaxies, including the Antennae. The right panel of Figure 14 presents the CMF/SFR statistic for NGC 3256 and shows that it is similar to that of the other seven galaxies. We measure the CMF/SFR statistic only for clusters younger than $\log (\tau /$ yr $)<7$, because our NGC 3256 sample does not contain a significant number of clusters older than this.

The uncertainties in the CMF/SFR statistic can be separated into uncertainties in the CMF and in the SFR value. Here we focus on the latter and refer the reader to Section 4.3.1, where we discussed uncertainties regarding the mass function. We adopt a total SFR of $50 M_{\odot} \mathrm{yr}^{-1}$ for NGC 3256 (Sakamoto et al. 2014), which was calculated from the bolometric infrared luminosity. This SFR is quite similar to the value of $46 M_{\odot} \mathrm{yr}^{-1}$ calculated from the total infrared luminosity and adopted in G10 for the SFR. We find other modern calculations of the SFR in NGC 3256 in a similar range, with a high value of 85 $M_{\odot} \mathrm{yr}^{-1}$ using infrared luminosity (Rodríguez-Zaurín et al. 2011). These values are consistent with an accuracy to within a factor of about two for the SFR, as found by several previous works (e.g., Lee et al. 2009; CFW15).

While our full area of coverage is $\approx 120 \mathrm{kpc}^{2}$, the clusters fall within an area of $\approx 50 \mathrm{kpc}^{2}$, comparable to that used for the SFR determinations mentioned above. While it is important that the same area of the galaxy be used for determining both the CMF and the SFR (as done in CFW15), this has not always been done. A number of studies have compared cluster properties with the SFR density of the host galaxy, but with the latter determined for a different area than for the clusters. For NGC 3256, G10 assumed a star-forming area of $\approx 75 \mathrm{kpc}^{2}$ (taken from the literature), but the actual area covered by their cluster catalog is only $\sim 33 \mathrm{kpc}^{2}$ (see Section 2.2 and Figure 10. We estimate the uncertainty in previously published values of the star-forming area to be approximately a factor of two for NGC 3256. This is on top of the uncertainty in the SFR itself.

CFW15 quantify the scatter in the CMF/SFR statistic among galaxies by fitting each galaxy by

$$
d N / d M=A \times \mathrm{SFR} \times\left(\mathrm{M} / 10^{4} \mathrm{M}_{\odot}\right)^{-1.9}
$$

and find the scatter in the normalization coefficient $A$ to be $\sigma(\log A)=0.21$. When NGC 3256 is added as an eighth galaxy, we find $\sigma(\log A)=0.24$.

If the formation of clusters is affected by the overall SFR or $\Sigma_{\text {SFR }}$ of their host galaxy, we should see a cor- relation of these properties with the residuals of $\log A$. In Figure 15. we plot the residuals of $\log A$ for each galaxy versus the SFR and $\Sigma_{\text {SFR }}$. We take $\Sigma_{\text {SFR }}$ values for all galaxies (including NGC 3256), but not the Antennae, from the recent compilation made by Adamo et al. (2015). The result for NGC 3256 is shown in red. The left panel is similar to the upper-left panel of Figure 4 in CFW15, who did not show the residuals with respect to $\Sigma_{\mathrm{SFR}}$. No obvious trend in the residuals is observed with either the SFR or with $\Sigma_{\text {SFR }}$. The nonparametric Spearman correlation coefficient, $r_{\mathrm{S}}$, and the corresponding probability $p\left(>r_{\mathrm{S}}\right)$ confirm this visual impression. We find $r_{\mathrm{S}}$ of -0.07 and $p\left(>r_{\mathrm{S}}\right)$ of 0.87 for $\log$ $A$ versus SFR and $r_{\mathrm{S}}$ of -0.24 and $p\left(>r_{\mathrm{S}}\right)$ of 0.57 for $\log A$ versus $\Sigma_{\mathrm{SFR}}$. These $p\left(>r_{\mathrm{S}}\right)$ values should be less than 0.05 to have a greater than $95 \%$ confidence of there being a correlation. Therefore, there is no statistically significant correlation between the residuals in $\log A$ and SFR and $\Sigma_{\text {SFR }}$ for these eight galaxies.

Our results for NGC 3256, when added to those for the seven galaxies presented in CFW15, are consistent with the hypothesis that the formation rates of stars and clusters are similar across star-forming galaxies that span a large range (approximately three orders of magnitude) in SFR and $\Sigma_{\text {SFR }}$. Our results for the age distribution in NGC 3256, which also shows a declining shape similar to that in the other more quiescent galaxies, suggest that the disruption history may also be the same, although this conclusion depends on the (currently unknown) SFH of the system. The cluster population models presented in Whitmore, Chandar, \& Fall (2007) indicate that for cluster disruption that is mostly independent of mass, the youngest clusters are the brightest. This is consistent with our findings for NGC 3256 in Section 3.2 .

\subsection{The $\Gamma$ Statistic}

A second, similar statistic that has been widely used in the literature is $\Gamma$, defined as the mass formed in bound clusters divided by the total stellar mass. The total mass in clusters, within a given age range, is typically found by extrapolating the observed CMF down to a mass of $100 M_{\odot}$. This extrapolation is accomplished either by assuming a power law or Schechter function, or by using a simulated cluster population. $\Gamma$ has been estimated for a number of galaxies and is typically plotted against $\Sigma_{\text {SFR }}$ of the host galaxy. Previously, both observational and theoretical works have suggested that there is a strong correlation between $\Gamma$ and $\Sigma_{\mathrm{SFR}}\left(\right.$ and $T_{L}(U)$ and $\Sigma_{\mathrm{SFR}}$ ), such that the higher the $\Sigma_{\mathrm{SFR}}$ of a galaxy, the higher the fraction of stars formed in bound clusters. Since estimates of $\Sigma_{\text {SFR }}$ and SFR correlate strongly for the specific galaxies studied thus far, there should be a similarly strong correlation between $\Gamma$ and SFR. If confirmed, this result would differ from the result of CFW15 who found little variation from galaxy to galaxy for CMF/SFR.

G10 estimated $\Gamma$ for NGC 3256, and found $\Gamma=$ $22.9 \%_{-9.8}^{+7.3}$. This was calculated from the CFR divided by the SFR, and it included only clusters with $\log (\tau / \mathrm{yr})<$ 7. We summarize their method below, and refer the reader to G10 for details. G10 found the total mass in clusters above a cutoff of $\log \left(M_{\text {cut }} / M_{\odot}\right)=4.7$ and used a synthetic cluster population to find the fraction of mass contained in clusters that is below this cutoff, as- 
suming a power law mass function with slope $\beta=-2.0$. They obtained the CFR by dividing their total mass in clusters by $7 \mathrm{Myr}$, rather than $10 \mathrm{Myr}$, due to the expectation that the clusters are embedded for the first 3 Myr and hence are missing from the sample. They divided the CFR by the SFR and obtained $\Gamma=0.12$. They made three additional corrections, each increasing $\Gamma$ by $\sim 25 \%$, giving a final value of $\Gamma=0.23$. G10 made these corrections in order to account for: (1) their rejection of clusters with poor fits and photometry, (2) a range of metallicities for young clusters in NGC 3256, which G10 found would increase the total cluster mass relative to their assumptions, and (3) an underestimate in actual cluster masses relative to derived masses.

We follow the G10 method, for the most part, to estimate $\Gamma$ from our larger cluster catalog in NGC 3256. We find the total mass in clusters above $\log \left(M_{\text {cut }} / M_{\odot}\right)=5.0$ and younger than $10 \mathrm{Myr}$ to be $7.23 \times 10^{7} M_{\odot}$. We use a similar synthetic cluster population as G10 to calculate the fraction of mass contained in clusters for $M\left(M>M_{\text {cut }}\right)$. We arrive at a CFR of $16.7 M_{\odot} \mathrm{yr}^{-1}$ by dividing $M_{\text {tot }}$ by $7 \mathrm{Myr}$, and we calculate $\Gamma=\mathrm{CFR} / \mathrm{SFR}$ $=0.33$, when we assume an SFR of $50 M_{\odot} \mathrm{yr}^{-1}$. We do not make the three $25 \%$ corrections in our determination of $\Gamma$, because our procedure does not result in the rejection of clusters with poor fits, we do not find any direct information on the metallicity distribution of young clusters in NGC 3256, and we are using different SSP models that, as far as we know, do not systematically underestimate cluster masses. Our $\Gamma$ value of 0.33 is 2.75 times higher than the value of 0.12 obtained by G10 before they make the additional corrections described above. This factor is the same as the relative offset in the masses that we found in Section 4.3. Our results suggest that $\Gamma$ is fairly robust when the same set of assumptions are used.

Ideally, $\Gamma$ should be determined using the same set of assumptions for all galaxies. However, this is not the current state of $\Gamma$ estimates in the literature, where different sets of assumptions have been used for different galaxies. For completeness, in Table 2, we compile different estimates of $\Gamma$ for NGC 3256 using different sets of assumptions. Here, column 4 shows our 'best' estimates of $\Gamma$, while column 5 includes the three $25 \%$ corrections used by G10 (labeled as $\Gamma^{\prime}$ ). We find that one of the biggest sources of uncertainty is the exact value used for the power law index of the CMF, $\beta$. G10 and other works have found that $\beta$ has typical uncertainties of the order of \pm 0.2 . However, changes at this level have a strong impact and change the derived value of $\Gamma$ by a factor of nearly four. $\Gamma$ is also sensitive to both the lower and upper mass ranges assumed in the extrapolation. If $\beta=-2.0$, then each decade in mass adds an equal percentage of total mass. For example, extrapolating down to a lower mass cutoff of, say, $1000 M_{\odot}$ rather than $100 M_{\odot}$ would increase the fraction of $M\left(M>M_{\text {cut }}\right)$ by $\sim 10 \%$. The estimates of $\Gamma$ vary from 0.09 to 0.98 (i.e., a factor of approximately 10!), depending on the specific choice of parameters. If we apply the same three $25 \%$ corrections as used by G10, we find a range between 0.18 and 1.91, i.e. the estimates go higher than $100 \%$.

As also found by G10, the exact value of $\Gamma$ is quite sensitive to the specific assumptions and extrapolations that are made. Because a variety of assumptions have been made for determinations of $\Gamma$ in different galaxies (which we will address in a forthcoming paper), and because this method requires more assumptions than the CMF/SFR statistic, the current determinations have larger uncertainties, which may make it more difficult to compare results between galaxies.

\section{SUMMARY AND CONCLUSIONS}

We have used ACS/WFC from HST to measure the properties of star clusters in the main body of NGC 3256. We draw the following conclusions.

1. The LF follows a power law with index $\alpha=-2.23 \pm$ 0.07 where $m_{V}<21.5$ for the sample combining inner (with $m_{V}<21.5$ ) and outer (with $m_{V}<23.0$ ) regions of NGC 3256. Measuring $\alpha$ for the inner and outer regions separately yielded $\alpha \approx-2.1$. These values agree with previous work.

2. We found that the age distribution can be described by a power law with index $\gamma \approx-0.67 \pm 0.08$ for independent mass ranges and for catalogs from the inner and outer areas of the galaxy separately. These values can be interpreted as a destruction rate of $\sim 80 \%$ each decade in time and are consistent with typical values of $\gamma=-0.8 \pm 0.2$ found in other systems.

3. The mass functions in various cluster age ranges are well described by a power law with index $\beta$. Young $(\log (\tau / \mathrm{yr})<7)$ clusters follow a robust $\beta=-1.86 \pm 0.34$. We found that $7<\log (\tau / \mathrm{yr})<8$ clusters are better described by $\beta=-1.31 \pm 0.36$, while $8<\log (\tau / \mathrm{yr})<8.6$ clusters follow $\beta=$ $-2.08 \pm 0.45$. We investigated a number of sources of uncertainty in $\beta$ and found that uncertainties agree with the formal uncertainties given in Figure 8

4. In order to test for the effect that image resolution can have on cluster properties, we artificially degraded an image of the Antennae and created independent catalogs from the degraded and original images. While less than half of the imagesmoothed sources were detected in the original image, the LFs, color distributions, and age distributions produced from both catalogs were very similar. We conclude that reliable measurement of the ages and luminosities of star clusters is not significantly hampered by the distance to NGC 3256 .

5. We considered two different methods that measure the efficiency with which clusters form in a galaxy. The CMF/SFR statistic was measured for eight galaxies, including NGC 3256, and had a dispersion of $\sigma(\log A)=0.24$. We measured $\Gamma$ and found a value of $33 \%$ from clusters younger than $10 \mathrm{Myr}$, and we discussed the different parameters and assumptions that affect this method.

We thank the referee for helpful comments. R.C. is grateful for support from NSF through CAREER award 0847467. This work is based on observations made with the NASA/ESA Hubble Space Telescope, and obtained 
from the Hubble Legacy Archive, which is a collaboration between the Space Telescope Science Institute (STScI/NASA), the Space Telescope European Coordinating Facility (ST-ECF/ESA) and the Canadian Astronomy Data Centre (CADC/NRC/CSA). This work was supported in part by NASA through grant GO-973501 from the Space Telescope Science Institute, which is operated by AURA, INC, under NASA contract NAS526555. This research has made use of the NASA/IPAC Extragalactic Database, which is operated by the Jet Propulsion Laboratory, California Institute of Technology, under contract with NASA.

\section{REFERENCES}

Adamo, A., Kruijssen, J. M. D., Bastian, N., Silva-Villa, E., \& Ryon, J. 2015, MNRAS, 452, 246

Adamo, A., Östlin, G., \& Zackrisson, E. 2011, MNRAS, 417, 1904

Bastian, N. 2008, MNRAS, 390, 759

Bastian, N., Adamo, A., Gieles, M., et al. 2012, MNRAS, 419, 2606

Baumgardt, H., Parmentier, G., Anders, P., \& Grebel, E. K. 2013, MNRAS, 430, 676

Bruzual, G. \& Charlot, S. 2003, MNRAS, 344, 1000

Chabrier, G. 2003, PASP, 115, 763

Chandar, R., Fall, S. M., \& Whitmore, B. C. 2010, ApJ, 711, 1263

Chandar, R., Fall, S. M., \& Whitmore, B. C. 2015, ApJ, 810, 1 (CFW15)

Chandar, R., Whitmore, B. C., Kim, H., et al. 2010, ApJ, 719, 966

Cook, D. O., Seth, A. C., Dale, D. A., et al. 2012, ApJ, 751, 100

Fall, S. M. \& Chandar, R. 2012, ApJ, 752, 96

Fall, S. M., Chandar, R., \& Whitmore, B. C. 2005, ApJ, 631, 133

Fouesneau, M., Johnson, L. C., Weisz, D. R., et al. 2014, ApJ, 786,117

Gieles, M. \& Portegies Zwart, S. F. 2011, MNRAS, 410, 6

Goddard, Q. E., Bastian, N., \& Kennicutt, R. C. 2010, MNRAS, 405, 857 (G10)
Harris, J. \& Zaritsky, D. 2004, AJ, 127, 1531

Harris, J. \& Zaritsky, D. 2009, AJ, 138, 1243

Hwang, N. \& Lee, M. G. 2010, ApJ, 709, 411

Karl, S. J., Fall, S. M., \& Naab T. 2011, ApJ, 734, 11

Knapen, J. H., Cisternas, M., \& Querejeta, M. 2015, 454, 1742

Knierman, K. A., Gallagher, S. C., Charlton, J. C. et al. 2003, AJ, 126, 1227

Kruijssen, J. M. D. 2012, MNRAS, 426, 3008

Kruijssen, J. M. D. 2014, CQGra, 31, 4006

Kruijssen, J. M. D. \& Bastian, N. 2016, MNRAS, 457, 24

Larsen, S. S. \& Richtler, T. 2000, A\&A, 354, 836

Lee, J. C., Gil de Paz, A., Tremonti, C., et al. 2009, ApJ, 706, 599

Maybhate, A., Masiero, J., Hibbard, J. E. et al. 2007, MNRAS, 381,59

Mulia, A. J., Chandar, R., \& Whitmore, B. C. 2015, ApJ, 805, 99

Randriamanakoto, Z., Väisänen, P., Ryder, S., Kankare, E., Kotilainen, J., \& Mattila, S. 2013, MNRAS, 431, 554

Rodríguez-Zaurín, J., Arribas, S. Monreal-Ibero, A., Colina, L., Alonso-Herrero, A., \& Alfonso-Garzón, J. 2011, A\&A, 527, 60

Sakamoto, K., Aalto, S., Combes, F., Evans, A., \& Peck, A. 2014, ApJ, 797, 90

Silva-Villa, E., Adamo, A., Bastian, N., Fouesneau, M., Zackrisson, E. 2014, MNRAS, 440,116

Silva-Villa, E., Larsen, S. S. 2011, A\&A, 529, 25

Sirianni, M., Jee, M. J., Benitez, N., et al. 2005, PASP, 117, 1049

Trancho, G., Bastian, N., Miller, B. W., \& Schweizer, F. 2007b, ApJ, 664, 284

Trancho, G., Bastian, N., Schweizer, F., \& Miller, B. W. 2007a, ApJ, 658, 993

Whitmore, B. C., Chandar, R., Bowers, A. S. et al. 2014, AJ, 147, 78

Whitmore, B. C., Chandar, R., Fall, S. M. 2007, AJ, 133, 1067

Whitmore, B. C., Zhang, Q., Leitherer, C., Fall, S. M., Schweizer, F., \& Miller, B. W. 1999, AJ, 118, 1551

Zepf, S. E., Ashman, K. M., English, J., Freeman, K. C., \&

Sharples, R. M. 1999, AJ, 118, 752

Zhang, Q. \& Fall, S. M. 1999, ApJ, 527, 81 


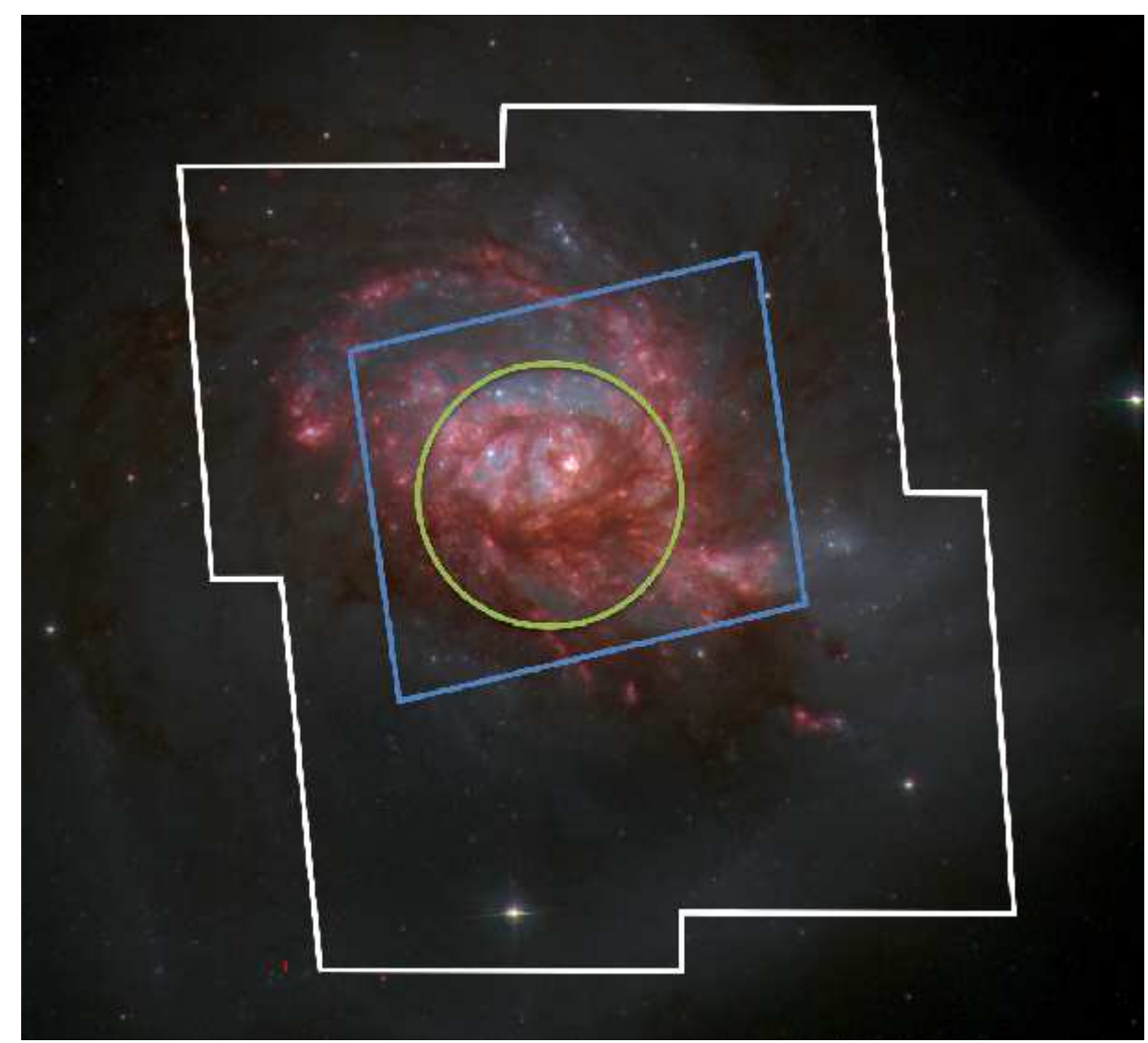

Figure 1. BVI color image of NGC 3256, with $\mathrm{H} \alpha$ overlaid. The green circle indicates where we divide our sample into inner and outer regions and is $\approx 9.5^{\prime \prime}(2 \mathrm{kpc})$ in radius. The area covered by G10 is shown as the blue rectangle and covers $\approx 3$ times less area than our coverage, shown as the white perimeter.

Table 1

Catalog of candidate star clusters in NGC 3256. Apparent magnitudes $\left(m_{\lambda}\right)$ and photometric errors $\left(\sigma_{\lambda}\right)$ are listed, as well as best-fit cluster ages in $\log$ years $(\tau)$, masses $(M)$ in $\log \left(M / M_{\odot}\right)$, and internal reddening $(E(B-V))$ as calculated from the SED fit. (This table is available in its entirety in machine-readable form.)

\begin{tabular}{|c|c|c|c|c|c|c|c|c|c|c|c|c|c|c|c|}
\hline ID & $\overline{\text { R.A. }}$ & Decl. & $\overline{m_{U}}$ & $\overline{\sigma_{U}}$ & $\overline{m_{B}}$ & $\overline{\sigma_{B}}$ & $\overline{m_{V}}$ & $\overline{\sigma_{V}}$ & $m_{H \alpha}$ & $\overline{\sigma_{H \alpha}}$ & $\overline{m_{I}}$ & $\overline{\sigma_{I}}$ & $\overline{\bar{M}}$ & $\bar{\tau}$ & $\overline{E(B-V)}$ \\
\hline 1 & 102752.352 & -435433.30 & 22.87 & 0.08 & 23.12 & 0.02 & 22.81 & 0.01 & 25.45 & 0.04 & 22.36 & 0.02 & 5.25 & 8.31 & 0.00 \\
\hline 2 & 102752.377 & -435432.71 & 23.16 & 0.11 & 23.13 & 0.02 & 22.90 & 0.01 & 54 & 5 & 22.30 & 0.02 & 5.25 & 8.41 & 0.00 \\
\hline 3 & 102749.477 & -435431.89 & 20.70 & 0.02 & 22.15 & 0.03 & 22.25 & 0.03 & 25.29 & 0.11 & 22.07 & 0.04 & 4.35 & 6.82 & 0.00 \\
\hline 4 & 102749.490 & -435431.77 & 20.58 & 0.03 & 21.81 & 0.04 & 21.79 & 0.03 & 24.75 & 0.09 & 21.56 & 0.04 & 4.56 & 6.84 & 0.00 \\
\hline 5 & 102749.626 & -435431.75 & 21.25 & 0.03 & 22.73 & 0.03 & 22.65 & 0.03 & 24.01 & 0.06 & 22.34 & 0.04 & 4.16 & 6.74 & 0.04 \\
\hline 6 & 102749.491 & -435431.44 & 19.40 & 0.01 & 20.81 & 0.02 & 20.87 & 0.02 & 23.89 & 0.04 & 20.58 & 0.02 & 4.93 & 6.84 & 0.00 \\
\hline 7 & 102749.724 & -435431.12 & 20.52 & 0.02 & 21.61 & 0.01 & 21.38 & 0.01 & 23.06 & 0.07 & 20.82 & 0.02 & 4.89 & 6.76 & 0.22 \\
\hline 8 & 102749.723 & -435431.00 & 20.33 & 0.01 & 21.53 & 0.02 & 21.35 & 0.02 & 23.24 & 0.11 & 20.76 & 0.02 & 4.95 & 6.86 & 0.14 \\
\hline 9 & 102750.821 & -435429.85 & 22.08 & 0.05 & 22.92 & 0.02 & 22.55 & 0.02 & 24.67 & 0.10 & 21.90 & 0.03 & 5.25 & 7.76 & 0.16 \\
\hline 10 & 102750.805 & -435429.81 & 22.70 & 0.10 & 23.37 & 0.05 & 22.75 & 0.04 & 25.47 & 0.33 & 21.97 & 0.05 & 5.46 & 8.01 & 0.28 \\
\hline
\end{tabular}




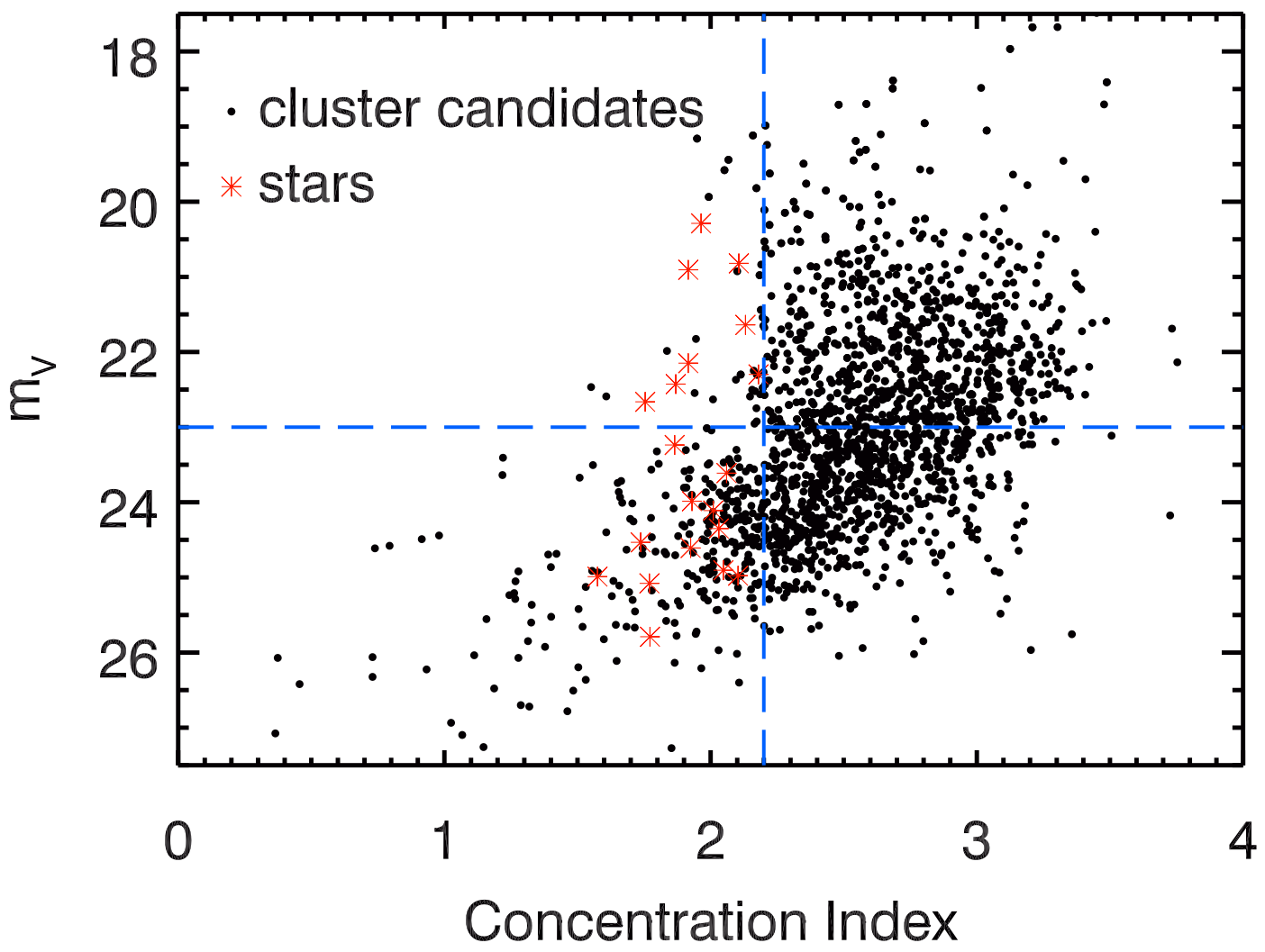

Figure 2. Plot of concentration index vs. brightness for all sources found in NGC 3256. The blue vertical line at $C=2.2$ illustrates that all likely field stars are more centrally concentrated than $C=2.2$. We note that the apparent relation between concentration index and brightness is an artifact of incompleteness and is not present above the horizontal line that illustrates our cutoff of $m_{V}=23.0$

Table 2

Different estimates of the fraction of stars forming in clusters $(\Gamma)$ in NGC 3256 using different assumptions. Column 4 shows $\Gamma$ with the given assumptions, while column 5 shows $\Gamma$ values when the three additional $25 \%$ correction factors used by G10 have been applied (see Section 6.2 for details).

\begin{tabular}{ccc|cc}
\hline \hline$\beta$ & SFR $\left(M_{\odot} \mathrm{yr}^{-1}\right)$ & $\Delta t(\mathrm{Myr})$ & $\Gamma$ & $\Gamma^{\prime}$ \\
\hline-1.8 & 46 & 7 & 0.24 & 0.47 \\
-1.8 & 46 & 10 & 0.17 & 0.33 \\
-1.8 & 50 & 7 & 0.22 & 0.43 \\
-1.8 & 50 & 10 & 0.15 & 0.30 \\
-1.8 & 85 & 7 & 0.13 & 0.25 \\
-1.8 & 85 & 10 & 0.09 & 0.18 \\
-2.0 & 46 & 7 & 0.36 & 0.71 \\
-2.0 & 46 & 10 & 0.25 & 0.50 \\
-2.0 & 50 & 7 & 0.33 & 0.65 \\
-2.0 & 50 & 10 & 0.23 & 0.46 \\
-2.0 & 85 & 7 & 0.20 & 0.38 \\
-2.0 & 85 & 10 & 0.14 & 0.27 \\
-2.2 & 46 & 7 & 0.98 & 1.91 \\
-2.2 & 46 & 10 & 0.68 & 1.33 \\
-2.2 & 50 & 7 & 0.90 & 1.75 \\
-2.2 & 50 & 10 & 0.63 & 1.23 \\
-2.2 & 85 & 7 & 0.53 & 1.03 \\
-2.2 & 85 & 10 & 0.37 & 0.72 \\
\hline
\end{tabular}




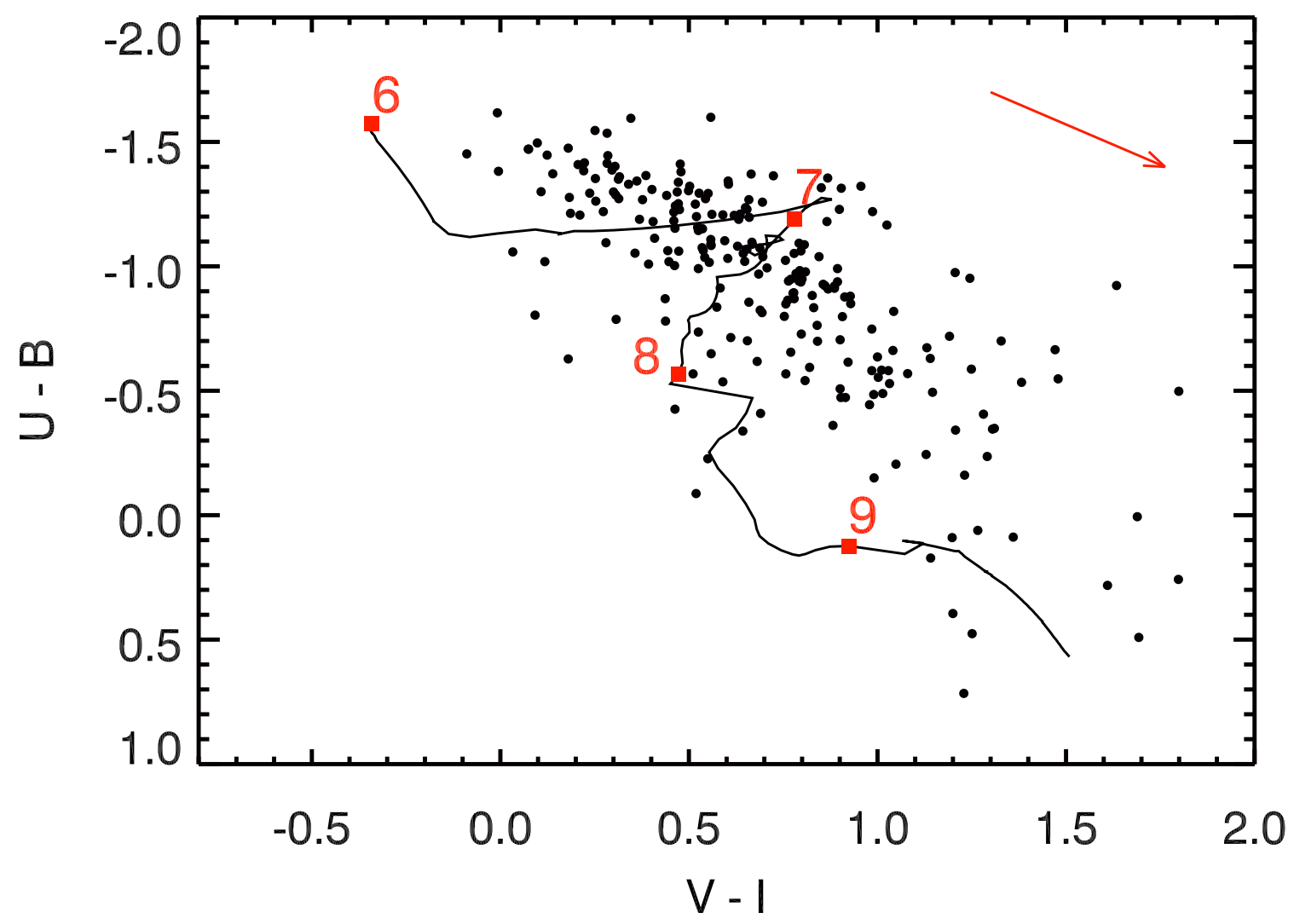

Figure 3. Color-color diagram of main body clusters in NGC 3256. For clarity, we show only the brightest clusters $\left(m_{V}<21.5\right)$. The red arrow shows the reddening vector for $A_{V}=1$. Various ages in $\log (\tau / \mathrm{yr})$ are shown by numbered red squares. 


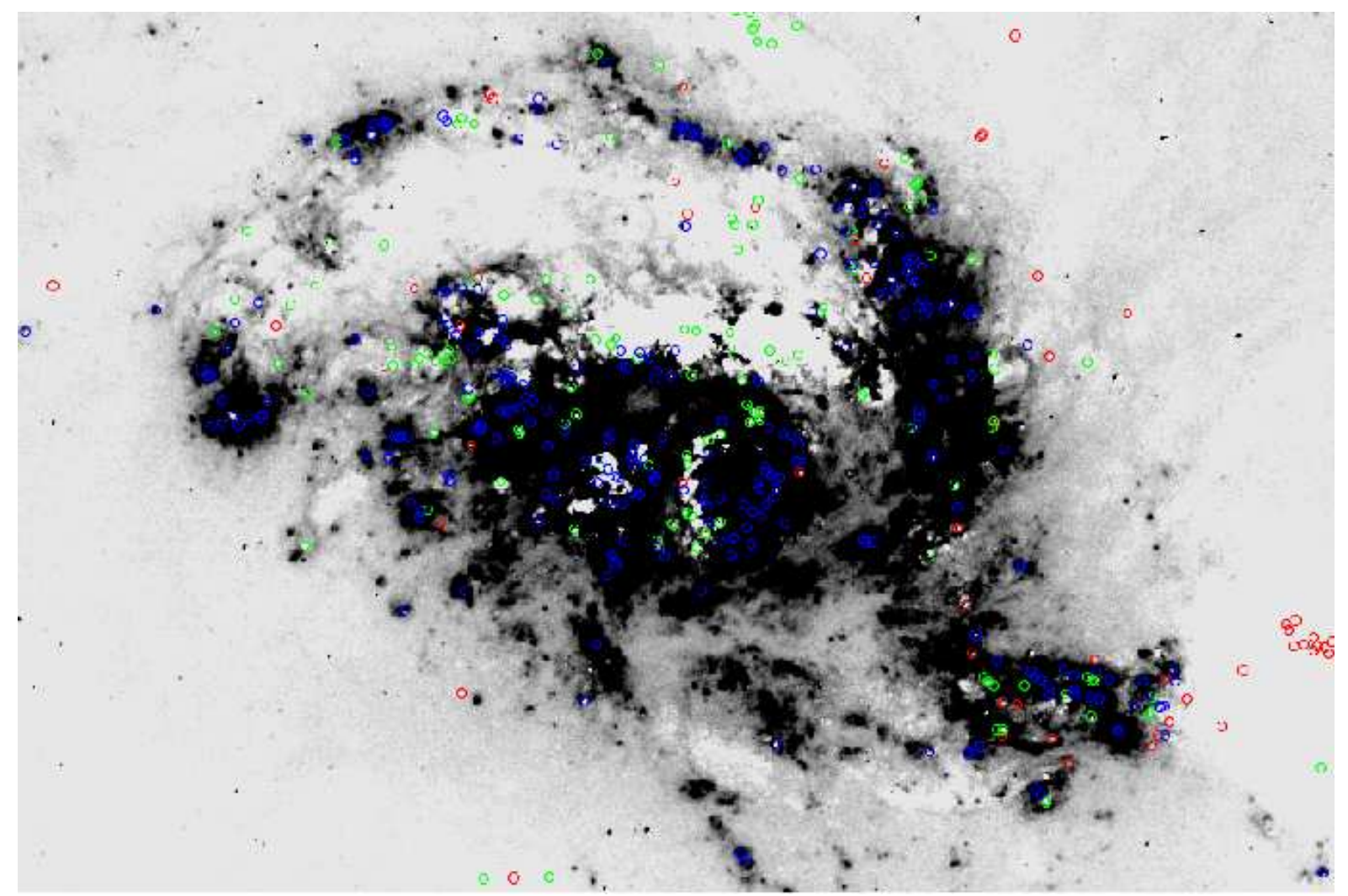

Figure 4. A map of $\mathrm{H} \alpha$ line emission, with continuum emission subtracted. The image has been inverted so that black regions show $\mathrm{H} \alpha$ line emission. Blue, green, and red circles indicate locations of clusters with ages $\log (\tau / \mathrm{yr})<7,7<\log (\tau / \mathrm{yr})<8$, and $8<\log (\tau / \mathrm{yr})<8.6$, respectively.

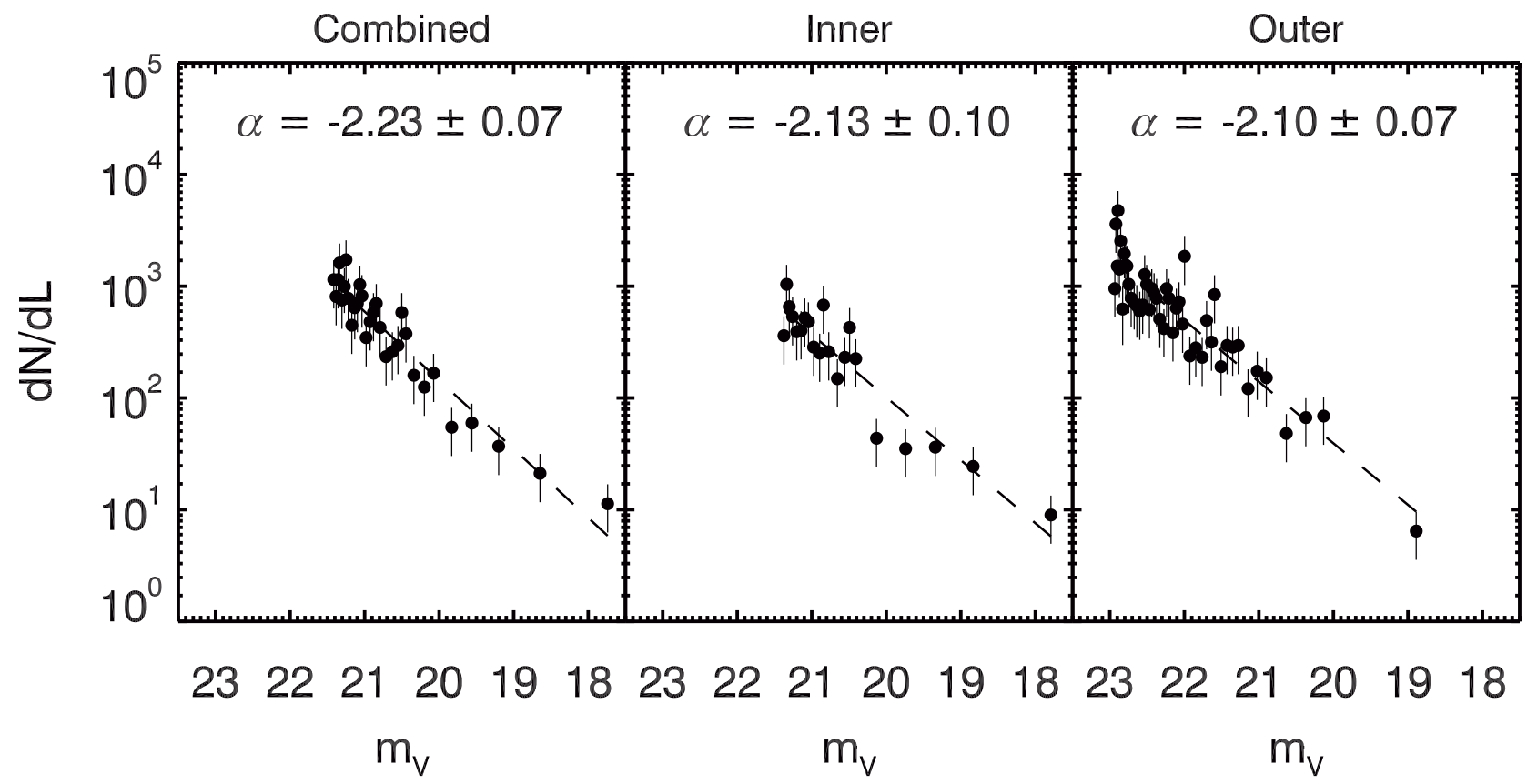

Figure 5. Luminosity functions of NGC 3256, uncorrected for internal extinction within NGC 3256 . On the left is the LF for all coverage of the main body. The middle panel shows the LF for the inner clusters, while the right panel shows the LF for the outer clusters. 


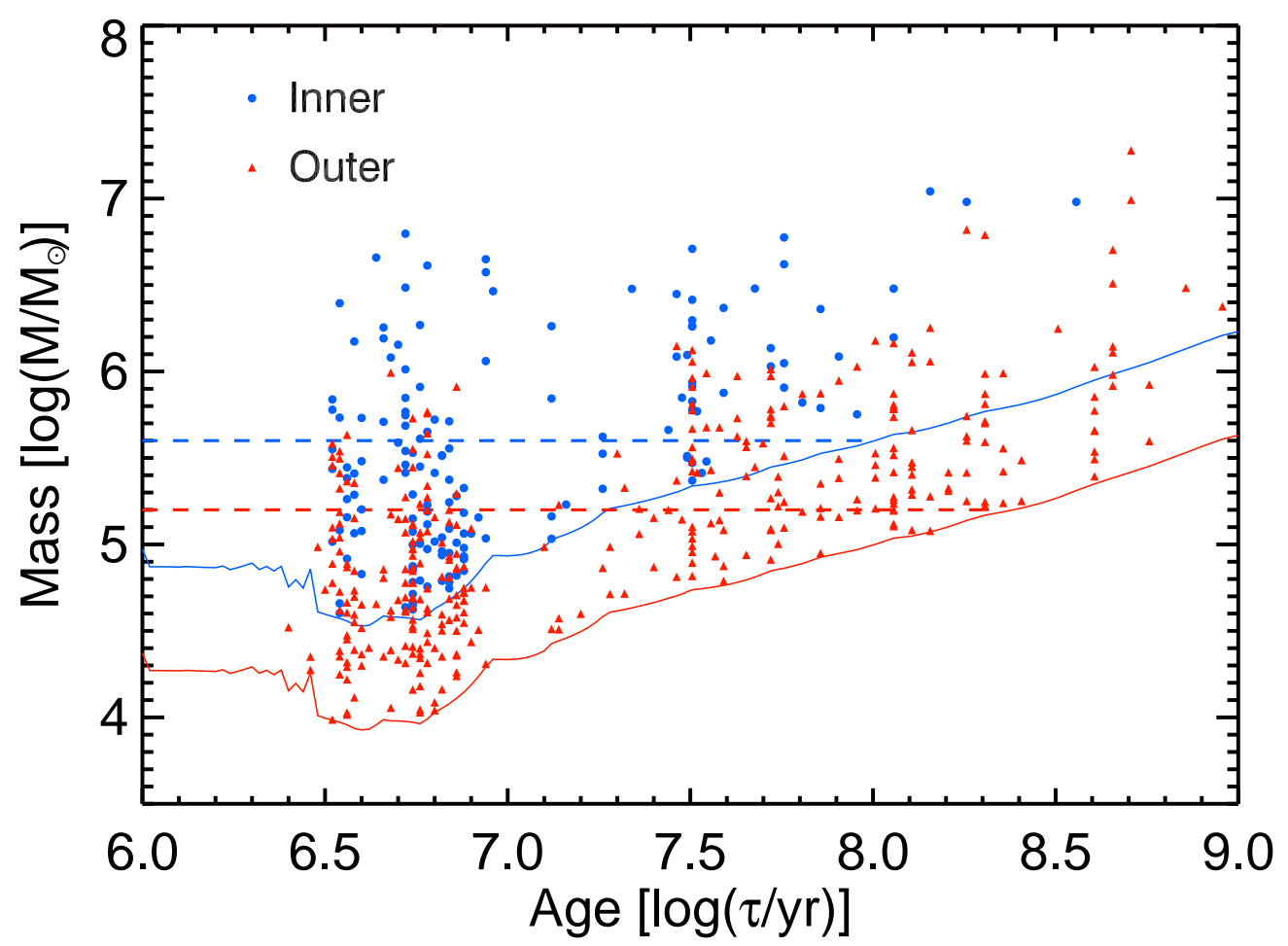

Figure 6. Mass-age relation for clusters in the inner and outer regions of NGC 3256. The solid lines illustrate our completeness limits of $m_{V}=21.5$ (blue) and $m_{V}=23.0$ (red) for the inner and outer catalogs, respectively.

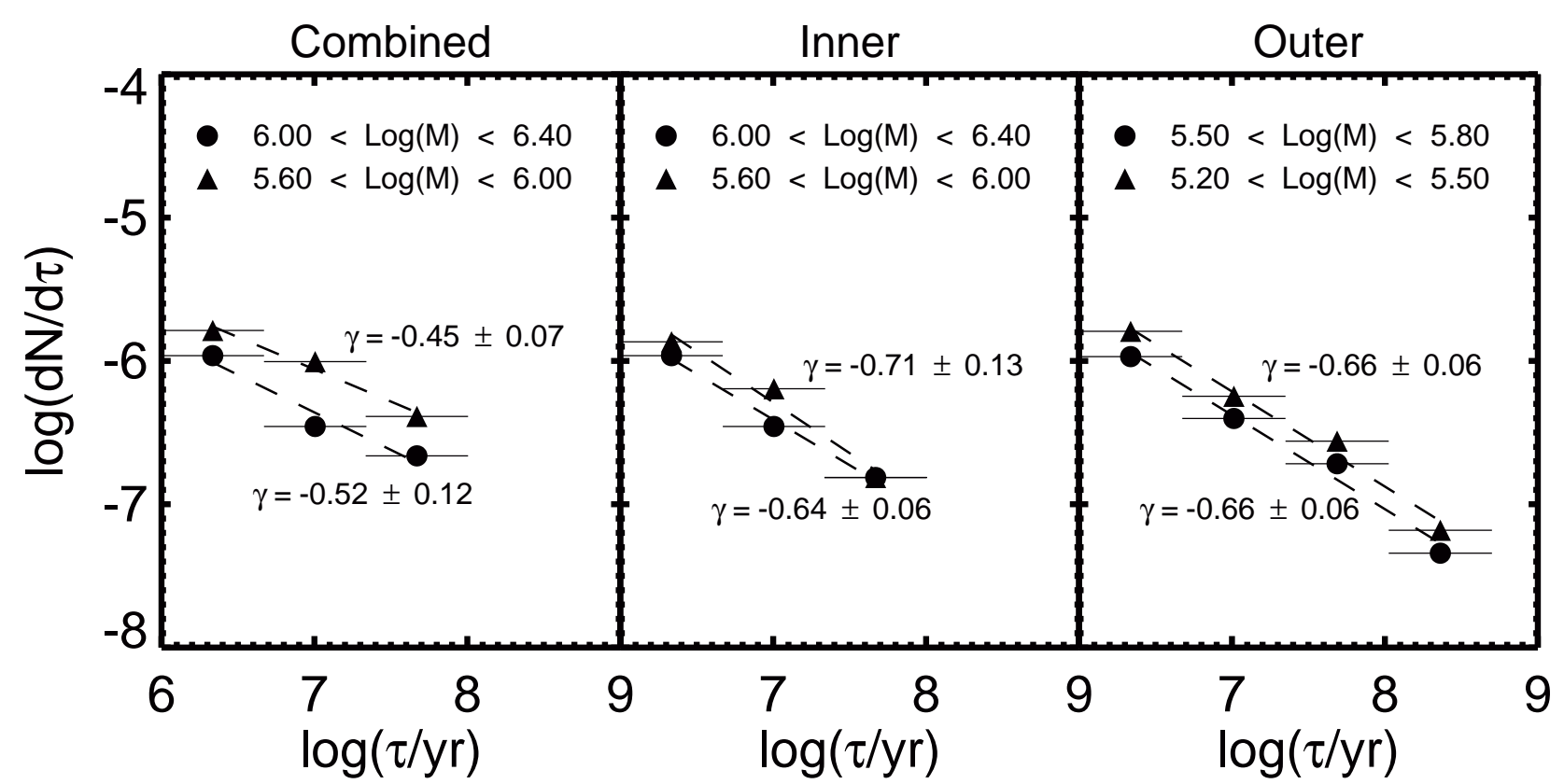

Figure 7. Age distributions of NGC 3256 for two mass regimes, with best fits shown as dashed lines. Different panels are for different catalogs. 


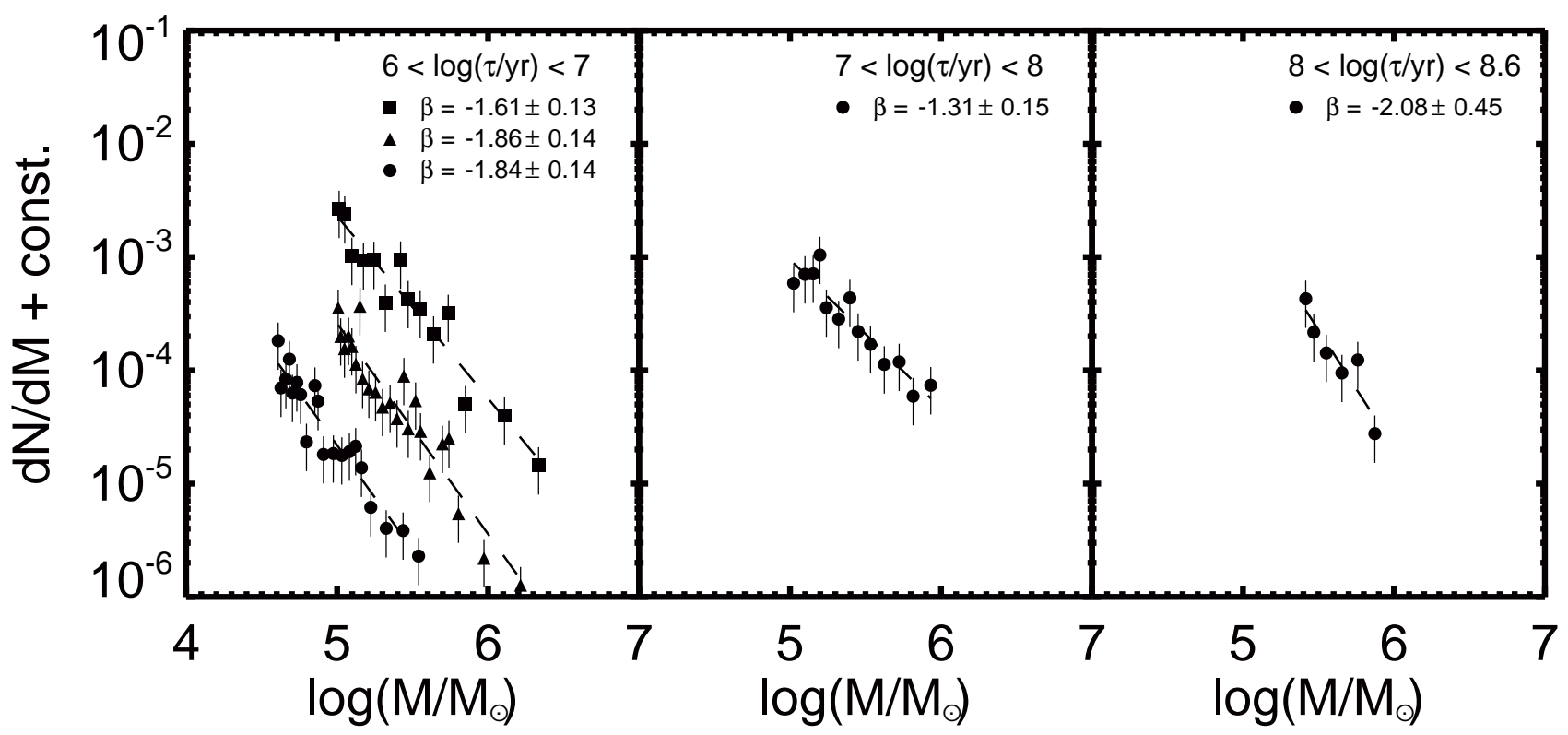

Figure 8. Cluster mass functions for NGC 3256 for three age ranges. Different symbols indicate catalogs from the inner area of the main body (squares), the outer area (circles), and both catalogs combined (triangles). The inner and combined catalogs contained too few clusters for reasonable fits to mass functions for ages $\log (\tau / \mathrm{yr})>7$ and are excluded from the middle and right panels. Power law fits to the mass functions are done for a constant number of clusters per bin. We find that a power law provides a good fit to the cluster mass functions in all cases. See Section 4.3 for details.

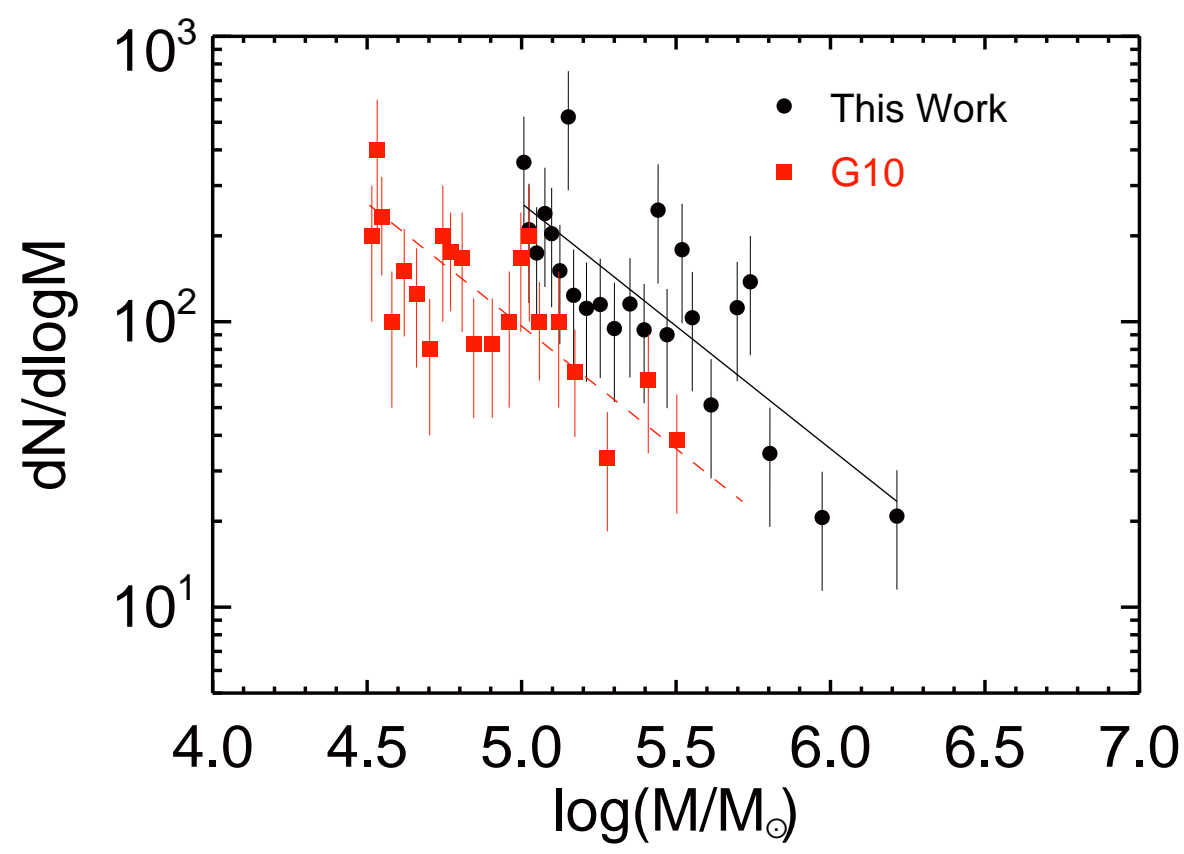

Figure 9. Cluster mass functions for $\log (\tau / \mathrm{yr})<7$ clusters in NGC 3256. The mass function constructed from the catalog from G10 is shown as red squares and compared to the mass function used in this work (shown in black). The black line shows the fit to our mass function. The dashed red line, which serves only as a visual guide to illustrate the horizontal offset, is the same as the solid black line but offset leftward by 0.5 , showing that the two mass functions have similar slopes. 


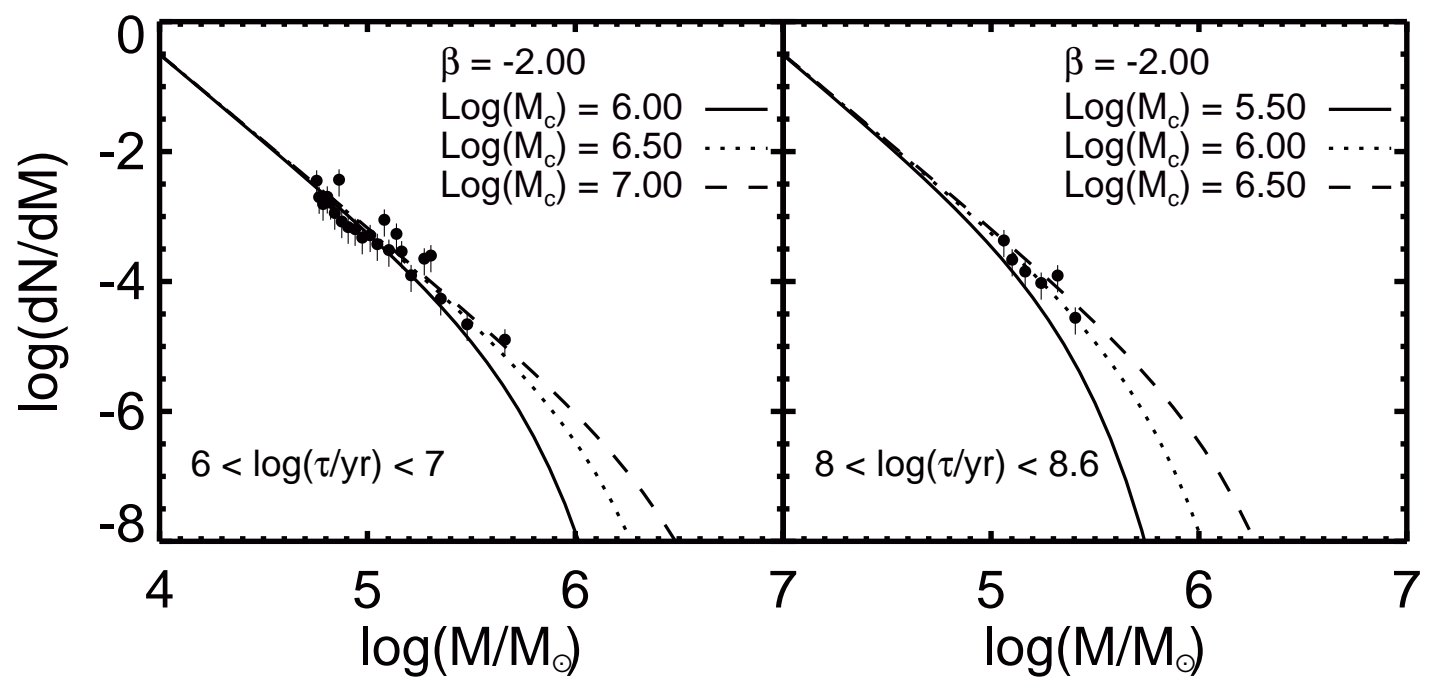

Figure 10. Cluster mass functions fit with Schechter functions, represented by $d N / d M \propto M^{\beta} \exp \left(-M / M_{C}\right)$. On the left, clusters in the range $6<\log (\tau /$ yr $)<7$ are well fit by $\beta=-2$ and $\log \left(M_{C}\right) \gtrsim 6.5$. On the right, the mass function for $8<\log (\tau /$ yr $)<8.6$ is fit by $\beta=-2$ and $\log \left(M_{C}\right) \gtrsim 6.0$. We find that the Schechter function is not necessary to fit the mass function (see Figure 8 ).
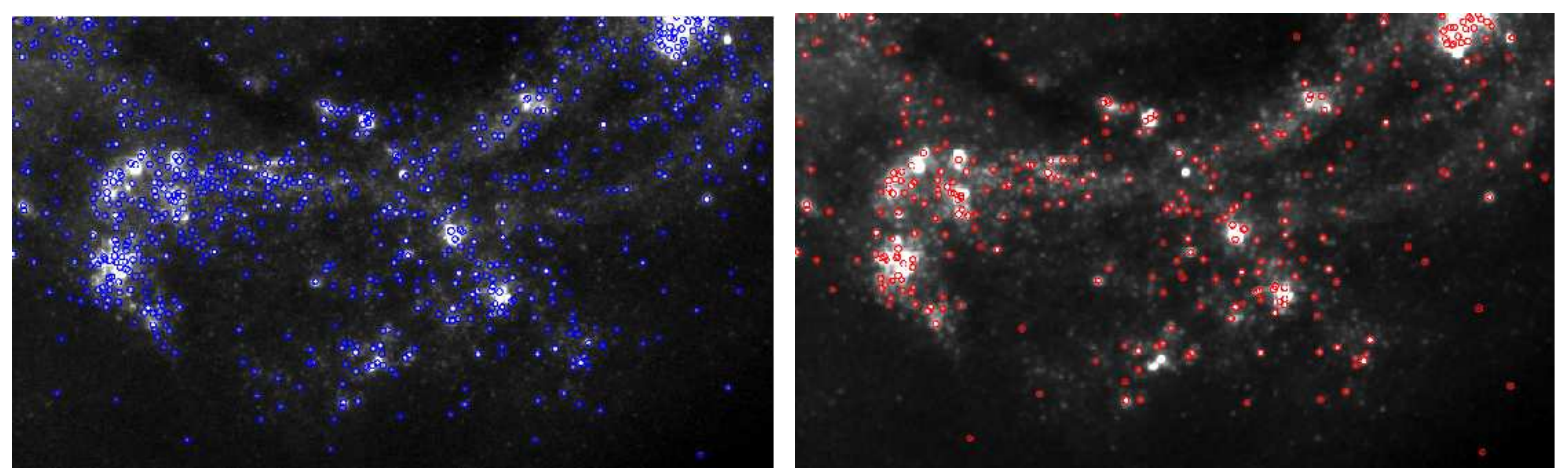

Figure 11. F550M images of a portion of the Antennae taken with $H S T /$ WFC at the native resolution $\left(0.05^{\prime \prime} /\right.$ pixel, left $)$ and smoothed by two pixels to simulate the distance of NGC 3256 (right). Circles indicate source detections from DAOFIND. By smoothing the image, many faint sources and sources in crowded areas are not detected. Despite this, the resulting LF and color distributions are quite similar. 


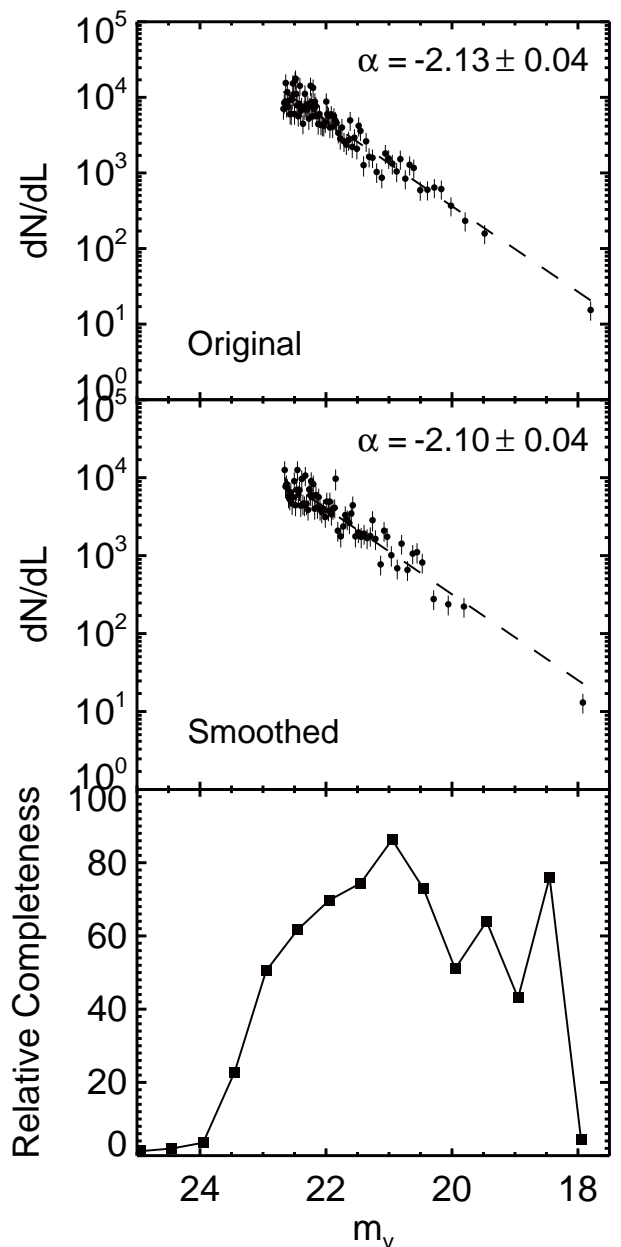

Figure 12. Top: the luminosity function for the Antennae galaxies based on clusters detected in the $V$ band image of $H S T$ /ACS. Middle: same as the top panel, but the LF resulting from detections made in the smoothed image. Note that nearly the same $\alpha$ value is obtained in both cases. Bottom: relative fraction of sources found in the image-smoothed catalog compared to the original catalog. 


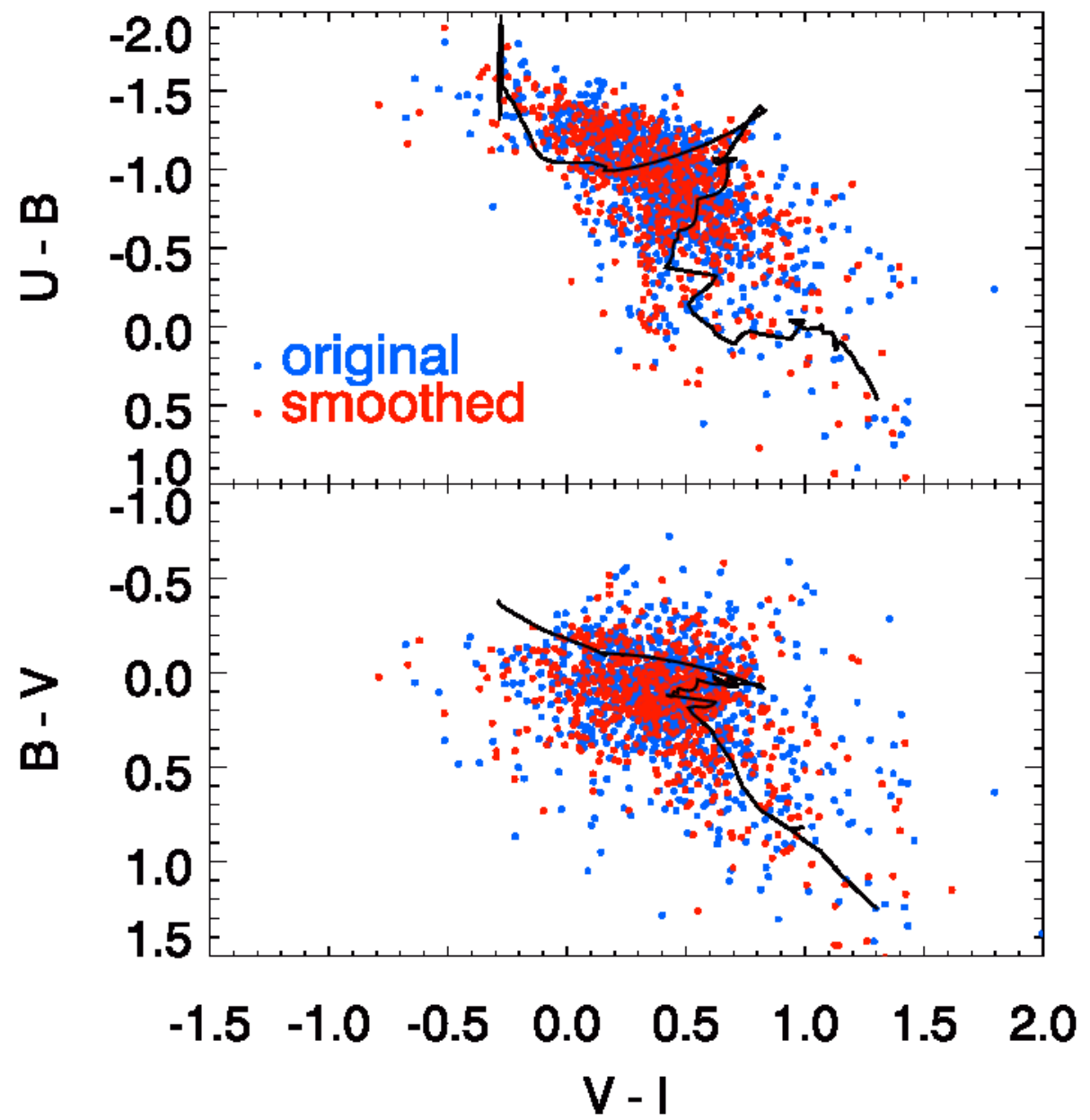

Figure 13. Color-color diagrams of the Antennae for clusters with $M_{V}<-9$. Blue and red points indicate colors from the original and image-smoothed catalogs, respectively.

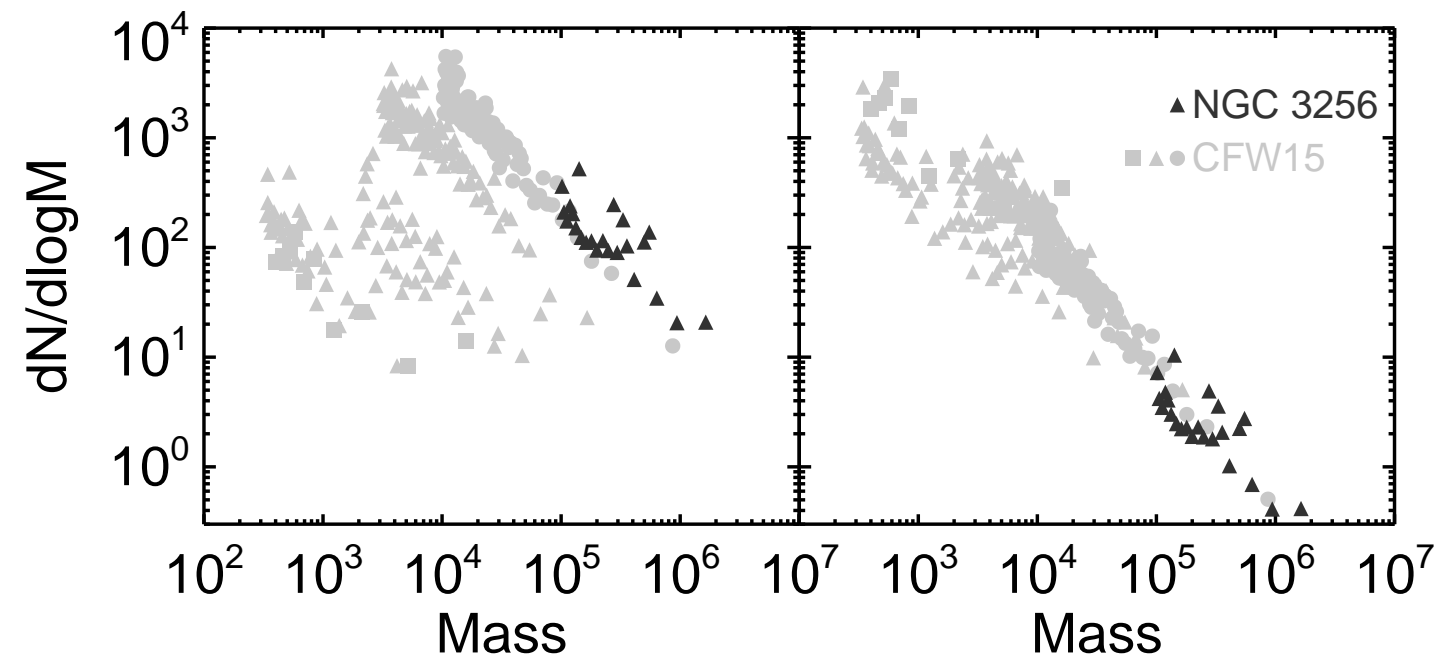

Figure 14. Left: the unnormalized mass function for NGC 3256 clusters younger than $\log (\tau / \mathrm{yr})<7$, shown in dark gray, while the lighter gray points show the unnormalized mass functions of seven other galaxies calculated in CFW15. Right: same as the left panel, with mass functions divided by the SFR of their host galaxy. NGC 3256 falls on top of and extends the CMF/SFR distributions in the other seven galaxies. 
18

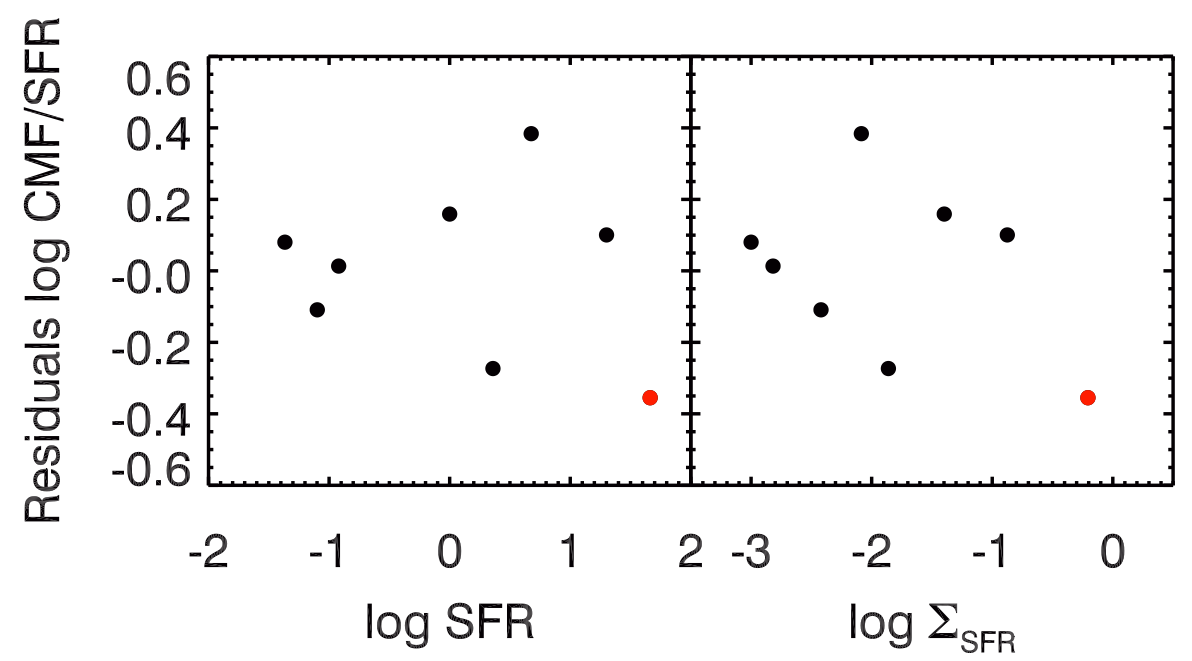

Figure 15. Residuals of CMF/SFR with respect to $\log (\mathrm{SFR})$ and $\log \left(\Sigma_{\mathrm{SFR}}\right)$, similar to Figure 4 of CFW15. The red point on both plots is for NGC 3256. No systematic trends are found. 